\title{
Drinfeld Twists and Symmetric Bethe Vectors of Supersymmetric Fermion Models
}

\author{
Shao-You Zhao ${ }^{1,2}$, Wen-Li Yang ${ }^{1,3}$ and Yao-Zhong Zhang ${ }^{1}$ \\ 1 Department of Mathematics, University of Queensland, Brisbane 4072, Australia \\ 2 Department of Physics, Beijing Institute of Technology, Beijing 100081, China \\ ${ }^{3}$ Institute of Modern Physics, Northwest University, Xi'an 710069, China
}

E-mail: syz@maths.uq.edu.au, wenli@maths.uq.edu.au, yzz@maths.uq.edu.au

\begin{abstract}
We construct the Drinfeld twists (factorizing $F$-matrices) of the $g l(m \mid n)$ invariant fermion model. Completely symmetric representation of the pseudoparticle creation operators of the model are obtained in the basis provided by the $F$-matrix (the $F$-basis). We resolve the hierarchy of the nested Bethe vectors in the $F$-basis for the $g l(m \mid n)$ supersymmetric model.
\end{abstract}

\section{Introduction}

In 1], it was realized that the $R$-matrices for the one-dimensional integrable XXX and XXZ spin chain systems are factorized in terms of certain non-degenerate lowertriangular F-matrices (Drinfeld twists [2])

$$
R_{12}\left(u_{1}, u_{2}\right)=F_{21}^{-1}\left(u_{2}, u_{1}\right) F_{12}\left(u_{1}, u_{2}\right)
$$

This leads to the natural $F$-basis for the analysis of these models. Working in the $F$-basis, the pseudo-particle creation operators of the systems take the completely symmetric form. Compared to the original Bethe vectors of the models, Bethe vectors in the $F$-basis are dramatically simplified and can be written down explicitly. These results allow form factors, correlation functions and spontaneous magnetizations of the systems to be represented in exact and compact form [3, 4].

The results of [1] were generalized to other models including the models associated with any finite-dimensional irreducible representations of the Yangian $Y[g l(2)]$ [5], the $g l(n)$ rational Heisenberg model [6], the elliptic XYZ and Belavin models [7, 8].

There are integrable models which do not come from a bosonic algebra. Examples include the Perk-Schultz model [9, whose exact solution and the Bethe ansatz equations were obtained in [10, 11, 12]. The actual algebra underlying the Perk-Schultz model is the Lie superalgebra $g l(m \mid n)$ : using the transformation introduced in [13], the $R$-matrix of the Perk-Schultz model is related to the $g l(m \mid n)$-invariant $R$-matrix. Integrable models with $g l(m \mid n)$ supersymmetry are physically important because they give strongly correlated fermion models of superconductivity. Interesting examples include the $g l(2 \mid 1)$-invariant supersymmetric $t-J$ model, the $g l(2 \mid 2)$-invariant electronic model [14] and the supersymmetric $U$ model [15], proposed in an attempt to understanding high- $T_{c}$ superconductivities. The application of the hierarchy of the algebraic Bethe ansatz to spin systems related to Lie superalgebras was given in [16]. 
Recently [17, 18, we have successfully constructed the Drinfeld twists for the $t$ - $J$ models with $g l(2 \mid 1)$ and $U_{q}(g l(2 \mid 1))$ supersymmetries, and resolved the hierarchies of the nested Bethe vectors of the two models. In this paper, we construct the factorizing $F$-matrices of the $g l(m \mid n)$-invariant fermion model. Working in the $F$-basis, we obtain the symmetric representations of the monodromy matrix and the creation operators. Moreover we resolve the hierarchy of the nested Bethe vectors in the $F$-basis for the $g l(m \mid n)$ model.

The present paper is organized as follows. In section II, we introduce some basic notation on the $g l(m \mid n)$-invariant fermion model. In section III, we construct the $F$ matrix and its inverse. In section IV, we give the representation of the monodromy matrix and creation operators in the $F$-basis. In section $\mathrm{V}$, specializing to the $g l(2 \mid 2)$ invariant superconductive electronic model, we resolve its nested Bethe vectors in the $F$-basis. In section VI, we resolve the hierarchy of the Bethe vectors of the $g l(m \mid n)$ model in the $F$-basis. We conclude the paper by offering some discussions in section VII.

\section{Basic definitions and notation}

Let $V$ be a $(m+n)$-dimensional $g l(m \mid n)$-module and $R \in \operatorname{End}(V \otimes V)$ be the $R$-matrix associated with this module. $V$ is $Z_{2}$-graded, and in the following we choose the following grading for $V:[1]=\ldots=[m]=1,[m+1]=\ldots=[m+n]=0$. The graded permutation operator $\mathcal{P}$ is defined by

$$
\mathcal{P}(a \otimes b)=(-1)^{[a][b]}(b \otimes a)
$$

or in matrix form

$$
(\mathcal{P})_{a b}^{c d}=(-1)^{[c][d]} \delta_{a d} \delta_{b c} .
$$

The $R$-matrix depends on the difference of two spectral parameters $u_{1}$ and $u_{2}$ associated with the two copies of $V$, and is given by

$$
R_{12}\left(u_{1}, u_{2}\right)=R_{12}\left(u_{1}-u_{2}\right)=a_{12} I+b_{12} \mathcal{P}
$$

where $I$ is the identity operator, and

$$
a_{12}=a\left(u_{1}, u_{2}\right) \equiv \frac{u_{1}-u_{2}}{u_{1}-u_{2}+\eta}, \quad b_{12}=b\left(u_{1}, u_{2}\right) \equiv \frac{\eta}{u_{1}-u_{2}+\eta}
$$

with $\eta \in C$ being the crossing parameter. One can easily check that the $R$-matrix satisfies the unitary relation

$$
R_{21} R_{12}=1
$$

Here and throughout $R_{12} \equiv R_{12}\left(u_{1}, u_{2}\right)$ and $R_{21} \equiv R_{21}\left(u_{2}, u_{1}\right)$. The $R$-matrix satisfies the graded Yang-Baxter equation (GYBE) 13

$$
R_{12} R_{13} R_{23}=R_{23} R_{13} R_{12}
$$


In terms of the matrix elements defined by

$$
R(u)\left(v^{i^{\prime}} \otimes v^{j^{\prime}}\right)=\sum_{i, j} R(u)_{i j}^{i^{\prime} j^{\prime}}\left(v^{i} \otimes v^{j}\right)
$$

the GYBE reads 13

$$
\begin{aligned}
& \sum_{i^{\prime}, j^{\prime}, k^{\prime}} R\left(u_{1}-u_{2}\right)_{i j}^{i^{\prime} j^{\prime}} R\left(u_{1}-u_{3}\right)_{i^{\prime} k}^{i^{\prime \prime} k^{\prime}} R\left(u_{2}-u_{3}\right)_{j^{\prime} k^{\prime}}^{j^{\prime \prime} k^{\prime \prime}}(-1)^{\left[j^{\prime}\right]\left(\left[i^{\prime}\right]+\left[i^{\prime \prime}\right]\right)} \\
= & \sum_{i^{\prime}, j^{\prime}, k^{\prime}} R\left(u_{2}-u_{3}\right)_{j k}^{j^{\prime} k^{\prime}} R\left(u_{1}-u_{3}\right)_{i k^{\prime}}^{i^{\prime} k^{\prime \prime}} R\left(u_{1}-u_{2}\right)_{i^{\prime} j^{\prime}}^{i^{\prime \prime} j^{\prime \prime}}(-1)^{\left[j^{\prime}\right]\left([i]+\left[i^{\prime}\right]\right)} .
\end{aligned}
$$

The quantum monodromy matrix $T(u)$ on a lattice of length $N$ is defined as

$$
T(u)=R_{0 N}\left(u, z_{N}\right) R_{0 N-1}\left(u, z_{N-1}\right) \ldots R_{01}\left(u, z_{1}\right),
$$

where the index 0 refers to the auxiliary space and $\left\{z_{i}\right\}$ are arbitrary inhomogeneous parameters depending on site $i$. $T(u)$ can be represented in the auxiliary space as a $(m+n) \times(m+n)$ matrix whose elements are operators acting on the quantum space $V^{\otimes N}$ :

$$
T(u)=\left(\begin{array}{cccc}
A_{11}(u) & \cdots & A_{1 m+n-1}(u) & B_{1}(u) \\
\vdots & \vdots & \vdots & \vdots \\
A_{m+n-11}(u) & \cdots & A_{m+n-1 m+n-1}(u) & B_{m+n-1}(u) \\
C_{1}(u) & \cdots & C_{m+n-1}(u) & D(u)
\end{array}\right)
$$

By using the GYBE, one may prove that the monodromy matrix satisfies the GYBE

$$
R_{12}(u-v) T_{1}(u) T_{2}(v)=T_{2}(v) T_{1}(u) R_{12}(u-v) .
$$

Define the transfer matrix $t(u)$

$$
t(u)=\operatorname{str}_{0} T(u)
$$

where $s t r_{0}$ denotes the supertrace over the auxiliary space. Then the Hamiltonian of the $g l(m \mid n)$ supersymmetric model is given by

$$
H=\left.\frac{d \ln t(u)}{d u}\right|_{u=0}
$$

This model is integrable thanks to the commutativity of the transfer matrix for different parameters,

$$
[t(u), t(v)]=0
$$

which can be verified by using the GYBE.

Following [1], we now introduce the notation $R_{1 \ldots N}^{\sigma}$, where $\sigma$ is any element of the permutation group $\mathcal{S}_{N}$. We note that we may rewrite the GYBE as

$$
R_{23}^{\sigma_{23}} T_{0,23}=T_{0,32} R_{23}^{\sigma_{23}}
$$


where $\sigma_{23}$ is the transposition of space labels $(2,3), R_{23}^{\sigma_{23}} \equiv R_{23}$, and $T_{0,23} \equiv R_{03} R_{02}$. It follows that $R_{1 \ldots N}^{\sigma}$ is a product of elementary $R$-matrices, corresponding to a decomposition of $\sigma$ into elementary transpositions. With the help of the GYBE, one may generalize (II.14) to a $N$-fold tensor product of spaces

$$
R_{1 \ldots N}^{\sigma} T_{0,1 \ldots N}=T_{0, \sigma(1 \ldots N)} R_{1 \ldots N}^{\sigma},
$$

where $T_{0,1 \ldots N} \equiv R_{0 N} \ldots R_{01}$. This implies the "decomposition" law

$$
R_{1 \ldots N}^{\sigma^{\prime} \sigma}=R_{\sigma^{\prime}(1 \ldots N)}^{\sigma} R_{1 \ldots N}^{\sigma^{\prime}}
$$

for a product of two elements in $\mathcal{S}_{N}$. We remark here that for the elementary transpo-

sition $\sigma_{i, i+1}, R_{1 \ldots N}^{\sigma_{i, i+1}}=R_{i, i+1}$, and for any $\sigma \in \mathcal{S}_{N}, R_{1 \ldots N}^{\sigma}$ can be obtained with the help of (II.16).

Note that $R_{\sigma^{\prime}(1 \ldots N)}^{\sigma}$ satisfies the relation

$$
R_{\sigma^{\prime}(1 \ldots N)}^{\sigma} T_{0, \sigma^{\prime}(1 \ldots N)}=T_{0, \sigma^{\prime} \sigma(1 \ldots N)} R_{\sigma^{\prime}(1 \ldots N)}^{\sigma} .
$$

As in [6], we write the elements of $R_{1 \ldots N}^{\sigma}$ as

$$
\left(R_{1 \ldots N}^{\sigma}\right)_{\beta_{N} \ldots \beta_{1}}^{\alpha_{\sigma(N) \ldots \alpha_{\sigma(1)}}}
$$

where the labels in the upper indices are permuted relative to the lower indices according to $\sigma$.

\section{F-matrices for the $g l(m \mid n)$ supersymmetric model}

In this section, we construct the factoring $F$-matrix and its inverse for the supersymmetric $g l(m \mid n)$ model.

\section{III.1 The F-matrix}

For the $R$-matrix (II.2), we define the $F$-matrix

$$
F_{12}=\sum_{m+n \geq \alpha_{2} \geq \alpha_{1}} P_{1}^{\alpha_{1}} P_{2}^{\alpha_{2}}+c_{12} \sum_{\gamma=1}^{m} P_{1}^{\gamma} P_{2}^{\gamma}+\sum_{m+n \geq \alpha_{1}>\alpha_{2}} P_{1}^{\alpha_{1}} P_{2}^{\alpha_{2}} R_{12}
$$

where $\left(P_{i}^{\alpha}\right)_{k}^{l}=\delta_{k, \alpha} \delta_{l, \alpha}$ is the projector acting on $i$ th space, and $c_{12} \equiv a_{12}-b_{12}$. Then by the $R$-matrix (II.2) and $F$-matrix (III.1), we have

$$
\begin{aligned}
F_{21} R_{12} & =\left(\sum_{m+n \geq \alpha_{1} \geq \alpha_{2}} P_{2}^{\alpha_{2}} P_{1}^{\alpha_{1}}+c_{21} \sum_{\gamma=1}^{m} P_{2}^{\gamma} P_{1}^{\gamma}+\sum_{m+n \geq \alpha_{2}>\alpha_{1}} P_{2}^{\alpha_{2}} P_{1}^{\alpha_{1}} R_{21}\right) R_{12} \\
& =\left(\sum_{m+n \geq \alpha_{1}>\alpha_{2}} P_{2}^{\alpha_{2}} P_{1}^{\alpha_{1}}+\left(1+c_{21}\right) \sum_{\gamma=1}^{m} P_{2}^{\gamma} P_{1}^{\gamma}+\sum_{\gamma=m+1}^{m+n} P_{2}^{\gamma} P_{1}^{\gamma}\right.
\end{aligned}
$$




$$
\begin{gathered}
\left.+\sum_{m+n \geq \alpha_{2}>\alpha_{1}} P_{2}^{\alpha_{2}} P_{1}^{\alpha_{1}} R_{21}\right) R_{12} \\
=\sum_{m+n \geq \alpha_{1}>\alpha_{2}} P_{2}^{\alpha_{2}} P_{1}^{\alpha_{1}} R_{12}+\left(1+c_{12}\right) \sum_{\gamma=1}^{m} P_{2}^{\gamma} P_{1}^{\gamma}+\sum_{\gamma=m+1}^{m+n} P_{2}^{\gamma} P_{1}^{\gamma} \\
+\sum_{m+n \geq \alpha_{2}>\alpha_{1}} P_{2}^{\alpha_{2}} P_{1}^{\alpha_{1}} \\
=\sum_{m+n \geq \alpha_{1}>\alpha_{2}} P_{2}^{\alpha_{2}} P_{1}^{\alpha_{1}} R_{12}+c_{12} \sum_{\gamma=1}^{m} P_{2}^{\gamma} P_{1}^{\gamma}+\sum_{m+n \geq \alpha_{2} \geq \alpha_{1}} P_{2}^{\alpha_{2}} P_{1}^{\alpha_{1}} \\
=F_{12} .
\end{gathered}
$$

Here we have used $R_{12} R_{21}=1$ and $c_{12} c_{21}=1$. Some remarks are in order. The solutions to (I.1), i.e. the $F$-matrices satisfying (I.1), are not unique [1, 6]. In this paper, we only consider particular solution of the form (III.1), which is lower-triangle.

We now generalize the $F$-matrix to the $N$-site problem. As is pointed out in [6], the generalized $F$-matrix should satisfy three properties: i) lower-triangularity; ii) nondegeneracy and

$$
\text { iii) } F_{\sigma(1 \ldots N)}\left(z_{\sigma(1)}, \ldots, z_{\sigma(N)}\right) R_{1 \ldots N}^{\sigma}\left(z_{1}, \ldots, z_{N}\right)=F_{1 \ldots N}\left(z_{1}, \ldots, z_{N}\right) \text {, }
$$

where $\sigma \in \mathcal{S}_{N}$ and $z_{i}, i=1, \ldots, N$, are generic inhomogeneous parameters .

Define the $N$-site $F$-matrix:

$$
F_{1, \ldots N}=\sum_{\sigma \in \mathcal{S}_{N}} \sum_{\alpha_{\sigma(1)} \ldots \alpha_{\sigma(N)}}^{*} \prod_{j=1}^{N} P_{\sigma(j)}^{\alpha_{\sigma(j)}} S\left(c, \sigma, \alpha_{\sigma}\right) R_{1 \ldots N}^{\sigma}
$$

where the sum $\sum^{*}$ is over all non-decreasing sequences of the labels $\alpha_{\sigma(i)}$ :

$$
\begin{array}{ll}
\alpha_{\sigma(i+1)} \geq \alpha_{\sigma(i)} & \text { if } \quad \sigma(i+1)>\sigma(i) \\
\alpha_{\sigma(i+1)}>\alpha_{\sigma(i)} & \text { if } \quad \sigma(i+1)<\sigma(i)
\end{array}
$$

and the c-number function $S\left(c, \sigma, \alpha_{\sigma}\right)$ is given by

$$
S\left(c, \sigma, \alpha_{\sigma}\right) \equiv \exp \left\{\sum_{l>k=1}^{N} \delta_{\alpha_{\sigma(k)}, \alpha_{\sigma(l)}}^{[\gamma]} \ln \left(1+c_{\sigma(k) \sigma(l)}\right)\right\}
$$

with $\gamma=1, \ldots, m, \delta_{\alpha_{\sigma(k)}, \alpha_{\sigma(l)}}^{[\gamma]}=1$ for $\alpha_{\sigma(k)}=\alpha_{\sigma(l)}=\gamma$, and $\delta_{\alpha_{\sigma(k)}, \alpha_{\sigma(l)}}^{[\gamma]}=0$ otherwise.

The definition of $F_{1 \ldots N}$, (III.4), and the summation condition (III.5) imply that $F_{1 \ldots N}$ is a lower-triangular matrix. Moreover, one can easily check that the $F$-matrix is non-degenerate because all diagonal elements are non-zero.

We now prove that the $F$-matrix (III.4) satisfies the property iii). Any given permutation $\sigma \in \mathcal{S}_{N}$ can be decomposed into elementary transpositions of the group $\mathcal{S}_{N}$ as $\sigma=\sigma_{1} \ldots \sigma_{k}$ with $\sigma_{i}$ denoting the elementary permutation $(i, i+1)$. By (II.16), we 
have if the property iii) holds for elementary transposition $\sigma_{i}$,

$$
\begin{aligned}
& F_{\sigma(1 \ldots N)} R_{1 \ldots N}^{\sigma}= \\
= & F_{\sigma_{1} \ldots \sigma_{k}(1 \ldots N)} R_{\sigma_{1} \ldots \sigma_{k-1}(1 \ldots N)}^{\sigma_{k}} R_{\sigma_{1} \ldots \sigma_{k-2}(1 \ldots N)}^{\sigma_{k-1}} \ldots R_{1 \ldots N}^{\sigma_{1}} \\
= & F_{\sigma_{1} \ldots \sigma_{k-1}(1 \ldots N)} R_{\sigma_{1} \ldots \sigma_{k-2}(1 \ldots N)}^{\sigma_{k-1}} \ldots R_{1 \ldots N}^{\sigma_{1}} \\
= & \ldots=F_{\sigma_{1}(1 \ldots N)} R_{1 \ldots N}^{\sigma_{1}}=F_{1 \ldots N} .
\end{aligned}
$$

For the elementary transposition $\sigma_{i}$, we have

$$
\begin{aligned}
& F_{\sigma_{i}(1 \ldots N)} R_{1 \ldots N}^{\sigma_{i}}= \\
= & \sum_{\sigma \in \mathcal{S}_{N}} \sum_{\alpha_{\sigma_{i} \sigma(1) \ldots \alpha_{\sigma_{i} \sigma(N)}}} \prod_{j=1}^{N} P_{\sigma_{i} \sigma(j)}^{\alpha_{\sigma_{i} \sigma(j)}} S\left(c, \sigma_{i} \sigma, \alpha_{\sigma_{i} \sigma}\right) R_{\sigma_{i}(1 \ldots N)}^{\sigma} R_{1 \ldots N}^{\sigma_{i}} \\
= & \sum_{\sigma \in \mathcal{S}_{N}} \sum_{\alpha_{\sigma_{i} \sigma(1) \ldots \alpha_{\sigma_{i} \sigma(N)}}}^{*} \prod_{j=1}^{N} P_{\sigma_{i} \sigma(j)}^{\alpha_{\sigma_{i} \sigma(j)}} S\left(c, \sigma_{i} \sigma, \alpha_{\sigma_{i} \sigma}\right) R_{1 \ldots N}^{\sigma_{i} \sigma} \\
= & \sum_{\tilde{\sigma} \in \mathcal{S}_{N}} \sum_{\alpha_{\tilde{\sigma}(1)} \ldots \alpha_{\tilde{\sigma}(N)}}^{*(i)} \prod_{j=1}^{N} P_{\tilde{\sigma}(j)}^{\alpha_{\tilde{\sigma}(j)}} S\left(c, \tilde{\sigma}, \alpha_{\tilde{\sigma}}\right) R_{1 \ldots N}^{\tilde{\sigma}},
\end{aligned}
$$

where $\tilde{\sigma}=\sigma_{i} \sigma$, and the summation sequences of $\alpha_{\tilde{\sigma}}$ in $\sum^{*(i)}$ now has the form

$$
\begin{array}{ll}
\alpha_{\tilde{\sigma}(j+1)} \geq \alpha_{\tilde{\sigma}(j)} & \text { if } \quad \sigma_{i} \tilde{\sigma}(j+1)>\sigma_{i} \tilde{\sigma}(j), \\
\alpha_{\tilde{\sigma}(j+1)}>\alpha_{\tilde{\sigma}(j)} & \text { if } \quad \sigma_{i} \tilde{\sigma}(j+1)<\sigma_{i} \tilde{\sigma}(j) .
\end{array}
$$

Comparing (III.9) with (III.5), we find that the only difference between them is the transposition $\sigma_{i}$ factor in the "if" conditions. For a given $\tilde{\sigma} \in \mathcal{S}_{N}$ with $\tilde{\sigma}(j)=i$ and $\tilde{\sigma}(k)=i+1$, one finds that if $|j-k|>1$, then $\sigma_{i}$ does not affect the sequence of $\alpha_{\tilde{\sigma}}$ at all, that is, the sign of inequality " $>$ " or " $\geq$ " between two neighboring root indexes is unchanged with the action of $\sigma_{i}$; and if $|j-k|=1$, then in the summation sequences of $\alpha_{\tilde{\sigma}}$, when $\tilde{\sigma}(j+1)=i+1$ and $\tilde{\sigma}(j)=i$, sign " $\geq$ " changes to " $>$ ", while when $\tilde{\sigma}(j+1)=i$ and $\tilde{\sigma}(j)=i+1$, "> " changes to " $\geq "$ ". Thus (III.5) and (III.8) differ only when equal labels $\alpha_{\tilde{\sigma}}$ appear. With the help of the $c$-number $S$ in the definition of $F_{1, \ldots, N}$ (III.4) and the relation $c_{12} c_{21}=1$, one easily shows that for the equal labels, $F_{\sigma_{i}(1 \ldots N)} R_{1 \ldots N}^{\sigma_{i}}-F_{1 \ldots N}=0$. (For detailed proof, please see [17].)

Therefore, we have proved that the Drinfeld twist factorizes the $R$-matrix of the $N$-site $g l(m \mid n)$ model:

$$
R_{1 \ldots N}^{\sigma}\left(z_{1}, \ldots, z_{N}\right)=F_{\sigma(1 \ldots N)}^{-1}\left(z_{\sigma(1)}, \ldots, z_{\sigma(N)}\right) F_{1 \ldots N}\left(z_{1}, \ldots, z_{N}\right) .
$$

In summary, the factorizing $F$-matrix $F_{1 \ldots N}$ of the $g l(m \mid n)$ model is proved to satisfy all three properties. 


\section{III.2 Inverse $F_{1 \ldots N}^{-1}$ of the $F$-matrix}

The non-degenerate property of the $F$-matrix implies that we can find the inverse matrix $F_{1 \ldots N}^{-1}$. To do so, we first define

$$
F_{1 \ldots N}^{*}=\sum_{\sigma \in \mathcal{S}_{N}} \sum_{\alpha_{\sigma(1)} \ldots \alpha_{\sigma(N)}}^{* *} S\left(c, \sigma, \alpha_{\sigma}\right) R_{\sigma(1 \ldots N)}^{\sigma^{-1}} \prod_{j=1}^{N} P_{\sigma(j)}^{\alpha_{\sigma(j)}}
$$

where the sum $\sum^{* *}$ is taken over all possible $\alpha_{i}$ which satisfies the following nonincreasing constraints:

$$
\begin{array}{ll}
\alpha_{\sigma(i+1)} \leq \alpha_{\sigma(i)} & \text { if } \quad \sigma(i+1)<\sigma(i) \\
\alpha_{\sigma(i+1)}<\alpha_{\sigma(i)} & \text { if } \quad \sigma(i+1)>\sigma(i)
\end{array}
$$

Now we compute the product of $F_{1 \ldots N}$ and $F_{1 \ldots N}^{*}$. Substituting (III.4) and (III.11) into the product, we have

$$
\begin{aligned}
F_{1 \ldots N} F_{1 \ldots N}^{*}= & \sum_{\sigma \in \mathcal{S}_{N}} \sum_{\sigma^{\prime} \in \mathcal{S}_{N}} \sum_{\alpha_{\sigma_{1}} \ldots \alpha_{\sigma_{N}}}^{*} \sum_{\beta_{\sigma_{1}^{\prime}} \ldots \beta_{\sigma_{N}^{\prime}}}^{* *} S\left(c, \sigma, \alpha_{\sigma}\right) S\left(c, \sigma^{\prime}, \beta_{\sigma^{\prime}}\right) \\
& \times \prod_{i=1}^{N} P_{\sigma(i)}^{\alpha_{\sigma(i)}} R_{1 \ldots N}^{\sigma} R_{\sigma^{\prime}(1 \ldots N)}^{\sigma^{\prime-1}} \prod_{i=1}^{N} P_{\sigma^{\prime}(i)}^{\beta_{\sigma^{\prime}(i)}} \\
= & \sum_{\sigma \in \mathcal{S}_{N}} \sum_{\sigma^{\prime} \in \mathcal{S}_{N}} \sum_{\alpha_{\sigma_{1} \ldots \alpha_{\sigma_{N}}}}^{*} \sum_{\sigma_{1}^{\prime} \ldots \beta_{\sigma_{N}^{\prime}}}^{* *} S\left(c, \sigma, \alpha_{\sigma}\right) S\left(c, \sigma^{\prime}, \beta_{\sigma^{\prime}}\right) \\
& \times \prod_{i=1}^{N} P_{\sigma(i)}^{\alpha_{\sigma(i)}} R_{\sigma^{\prime}(1 \ldots N)}^{\sigma^{\prime-1} \sigma} \prod_{i=1}^{N} P_{\sigma^{\prime}(i)}^{\beta_{\sigma^{\prime}(i)}} .
\end{aligned}
$$

To evaluate the r.h.s., we examine the matrix element of the $R$-matrix

$$
\left(R_{\sigma^{\prime}(1 \ldots N)}^{\sigma^{\prime-1} \sigma}\right)_{\beta_{\sigma^{\prime}(N) \ldots \beta_{\sigma^{\prime}(1)}}}^{\alpha_{\sigma(N) \ldots \alpha_{\sigma(1)}}}
$$

Note that the sequence $\left\{\alpha_{\sigma}\right\}$ is non-decreasing and $\left\{\beta_{\sigma^{\prime}}\right\}$ is non-increasing. Thus the non-vanishing condition of the matrix element (III.14) requires that $\alpha_{\sigma}$ and $\beta_{\sigma^{\prime}}$ satisfy

$$
\beta_{\sigma^{\prime}(N)}=\alpha_{\sigma(1)}, \ldots, \beta_{\sigma^{\prime}(1)}=\alpha_{\sigma(N)} .
$$

One can verify [6] that (III.15) is fulfilled only if

$$
\sigma^{\prime}(N)=\sigma(1), \ldots, \sigma^{\prime}(1)=\sigma(N) .
$$

Let $\bar{\sigma}$ be the maximal element of the $\mathcal{S}_{N}$ which reverses the site labels

$$
\bar{\sigma}(1, \ldots, N)=(N, \ldots, 1)
$$


Then from (III.16), we have

$$
\sigma^{\prime}=\sigma \bar{\sigma}
$$

Substituting (III.15) and (III.18) into (III.13), we have

$$
F_{1 \ldots N} F_{1 \ldots N}^{*}=\sum_{\sigma \in \mathcal{S}_{N}} \sum_{\alpha_{\sigma_{1}} \ldots \alpha_{\sigma_{N}}}^{*} S\left(c, \sigma, \alpha_{\sigma}\right) S\left(c, \sigma, \alpha_{\sigma}\right) \prod_{i=1}^{N} P_{\sigma(i)}^{\alpha_{\sigma(i)}} R_{\sigma(N \ldots 1)}^{\bar{\sigma}} \prod_{i=1}^{N} P_{\sigma(i)}^{\alpha_{\sigma(i)}} .
$$

The decomposition of $R^{\bar{\sigma}}$ in terms of elementary $R$-matrices is unique module GYBE. One reduces from (III.19) that $F F^{*}$ is a diagonal matrix:

$$
F_{1 \ldots N} F_{1 \ldots N}^{*}=\prod_{i<j} \Delta_{i j}
$$

where

$$
\left[\Delta_{i j}\right]_{\alpha_{i} \alpha_{j}}^{\beta_{i} \beta_{j}}=\delta_{\alpha_{i} \beta_{i}} \delta_{\alpha_{j} \beta_{j}}\left\{\begin{array}{cl}
a_{i j} & \text { if } \alpha_{i}>\alpha_{j} \\
a_{j i} & \text { if } \alpha_{i}<\alpha_{j} \\
4 a_{i j} a_{j i} & \text { if } \alpha_{i}=\alpha_{j}=1,2, \ldots, m \\
1 & \text { if } \alpha_{i}=\alpha_{j}=m+1, \ldots, m+n
\end{array}\right.
$$

Therefore, the inverse of the $F$-matrix is given by

$$
F_{1 \ldots N}^{-1}=F_{1 \ldots N}^{*} \prod_{i<j} \Delta_{i j}^{-1}
$$

\section{The monodromy matrix in the $F$-basis}

In the previous section, we see that the $g l(m \mid n) R$-matrix factorizes in terms of the $F$-matrix and its inverse which we constructed explicitly. The column vectors of the inverse of the $F$-matrix form a set of basis on which $g l(m \mid n)$ acts. In this section, we study the generators of $g l(m \mid n)$ and the elements of the monodromy matrix in the $F$-basis.

\section{IV.1 $g l(m \mid n)$ generators in the $F$-basis}

Denoted by $E^{\gamma}{ }^{\gamma \pm 1}$ the simple generators of the $N$-site $g l(m \mid n)$ supersymmetric system. Then $E^{\gamma \gamma \pm 1}=E_{(1)}^{\gamma \gamma \pm 1}+\ldots+E_{(N)}^{\gamma \gamma \pm 1}$, where $E_{(k)}^{\gamma \gamma \pm 1}$ acts on the $k$ th component of the tensor product space. Let $\tilde{E}^{\gamma \gamma \pm 1}$ denote the corresponding simple generators in the $F$ basis: $\tilde{E}^{\gamma \gamma \pm 1}=F_{1 \ldots N} E^{\gamma \gamma \pm 1} F_{1 \ldots N}^{-1}$. For later use, we derive $\tilde{E}^{\gamma \gamma+1}$. From the expressions of $F$ and its inverse, we have

$$
\begin{aligned}
\tilde{E}^{\gamma \gamma+1} & =F_{1 \ldots N} E^{\gamma \gamma+1} F_{1 \ldots N}^{-1} \\
& =\sum_{\sigma, \sigma^{\prime} \in \mathcal{S}_{N}} \sum_{\alpha_{\sigma(1)} \ldots \alpha_{\sigma(N)}}^{*} \sum_{\beta_{\sigma^{\prime}(1)} \ldots \beta_{\sigma^{\prime}(N)}}^{* *} S\left(c, \sigma, \alpha_{\sigma}\right) S\left(c, \sigma^{\prime}, \beta_{\sigma^{\prime}}\right)
\end{aligned}
$$




$$
\begin{aligned}
& \times \prod_{i=1}^{N} P_{\sigma(i)}^{\alpha_{\sigma(i)}} R_{1 \ldots N}^{\sigma} E^{\gamma \gamma+1} R_{\sigma^{\prime}(1 \ldots N)}^{\sigma^{\prime-1}} \prod_{i=1}^{N} P_{\sigma^{\prime}(i)}^{\beta_{\sigma^{\prime}(i)}} \prod_{i<j} \Delta_{i j}^{-1} \\
& =\sum_{\sigma, \sigma^{\prime} \in \mathcal{S}_{N}} \sum_{\alpha_{\sigma(1)} \ldots \alpha_{\sigma(N)}}^{*} \sum_{\beta_{\sigma^{\prime}(1)} \ldots \beta_{\sigma^{\prime}(N)}}^{* *} S\left(c, \sigma, \alpha_{\sigma}\right) S\left(c, \sigma^{\prime}, \beta_{\sigma^{\prime}}\right) \\
& \times \prod_{i=1}^{N} P_{\sigma(i)}^{\alpha_{\sigma(i)}} E^{\gamma \gamma+1} R_{\sigma^{\prime}(1 \ldots N)}^{\sigma^{\prime-1} \sigma} \prod_{i=1}^{N} P_{\sigma^{\prime}(i)}^{\beta_{\sigma^{\prime}(i)}} \prod_{i<j} \Delta_{i j}^{-1} \\
& =\sum_{\sigma, \sigma^{\prime} \in \mathcal{S}_{N}} \sum_{k=1}^{N} E_{(\sigma(a+l))}^{\gamma \gamma+1} \sum_{\alpha_{\sigma(1)} \ldots \alpha_{\sigma(N)}}^{*} \sum_{\beta_{\sigma^{\prime}(1)} \ldots \beta_{\sigma^{\prime}(N)}}^{* *} S\left(c, \sigma, \alpha_{\sigma}\right) S\left(c, \sigma^{\prime}, \beta_{\sigma^{\prime}}\right) \\
& \times P_{\sigma(1)}^{\alpha_{\sigma(1)}} P_{\sigma(2)}^{\alpha_{\sigma(2)}} \ldots P_{\sigma(a)}^{\alpha_{\sigma(a)}=\gamma-1} P_{\sigma(a+1)}^{\alpha_{\sigma(a+1)}=\gamma} \ldots\left(P_{\sigma(a+l)=k}^{\alpha_{\sigma(a+l)}=\gamma \rightarrow \gamma+1}\right) \ldots \\
& \times P_{\sigma(N)}^{\alpha_{\sigma(N)}} R_{\sigma^{\prime}(1 \ldots N)}^{\sigma^{\prime-1} \sigma} \prod_{i=1}^{N} P_{\sigma^{\prime}(i)}^{\beta_{\sigma^{\prime}(i)}} \prod_{i<j} \Delta_{i j}^{-1},
\end{aligned}
$$

where in (IV.1), we have used $\left[E^{\gamma \gamma \pm 1}, R_{1 \ldots N}^{\sigma}\right]=0$. The element of $R_{\sigma^{\prime}(1 \ldots N)}^{\sigma^{\prime-1} \sigma}$ between $P_{\sigma(1)}^{\alpha_{\sigma(1)}} \ldots\left(P_{\sigma(a+l)=k}^{\alpha_{\sigma(a+l)}=\gamma \rightarrow \gamma+1}\right) \ldots P_{\sigma(N)}^{\alpha_{\sigma(N)}}$ and $P_{\sigma^{\prime}(N)}^{\beta_{\sigma^{\prime}(N)}} \ldots P_{\sigma^{\prime}(1)}^{\beta_{\sigma^{\prime}(1)}}$ is denoted as

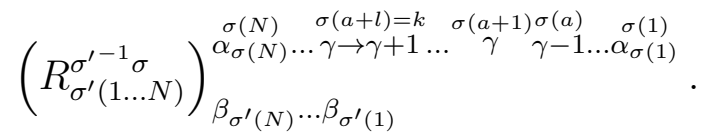

We call the sequence $\left\{\alpha_{\sigma(l)}\right\}$ normal if it is arranged according to the rules in (III.5), otherwise, we call it abnormal.

It is now convenient for us to discuss the non-vanishing condition of the $R$-matrix element (IV.3) . Comparing (IV.3) with (III.14), we find that the difference between them lies in the $k$ th site. Because the group label in the $k$ th space has been changed, the sequence $\left\{\alpha_{\sigma}\right\}$ is now a abnormal sequence. However, it can be permuted to the normal sequence by some permutation $\hat{\sigma}_{k}$. Namely, $\alpha_{\gamma \rightarrow \gamma+1}$ in the abnormal sequence can be moved to a suitable position by using the permutation $\hat{\sigma}_{k}$ according to rules in (III.5). (It is easy to verify that $\hat{\sigma}_{k}$ is unique by using (III.5).) Thus, by procedure similar to that in the previous section, we find that when

$$
\sigma^{\prime}=\hat{\sigma}_{k} \sigma \bar{\sigma} \quad \text { and } \quad \beta_{\sigma^{\prime}(N)}=\alpha_{\sigma(1)}, \ldots, \beta_{\sigma^{\prime}(1)}=\alpha_{\sigma(N)}
$$

the $R$-matrix element (IV.3) is non-vanishing.

Because the non-zero condition of the elementary $R$-matrix element $R_{i j}^{i^{\prime} j^{\prime}}$ is $i+j=$ $i^{\prime}+j^{\prime}$, the following $R$-matrix elements

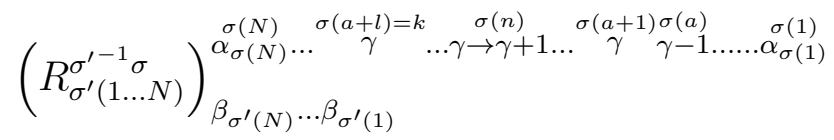

with $a+1 \leq n<a+l$ are also non-vanishing. 
Therefore, (IV.2) becomes

$$
\begin{aligned}
\tilde{E}^{\gamma \gamma+1}= & \sum_{\sigma \in \mathcal{S}_{N}} \sum_{k=1}^{N} \sum_{\alpha_{\sigma_{1}} \ldots \alpha_{\sigma_{N}}}^{*} S\left(c, \sigma, \alpha_{\sigma}\right) S\left(c, \hat{\sigma}_{k} \sigma, \alpha_{\hat{\sigma}_{k} \sigma}\right) \\
& \times\left[E_{(\sigma(a+l))}^{\gamma \gamma+1} P_{\sigma(1)}^{\alpha_{\sigma(1)}} \ldots P_{\sigma(a)}^{\gamma-1} P_{\sigma(a+1)}^{\gamma} \ldots P_{\sigma(a+l)=k}^{\gamma \rightarrow \gamma+1} \ldots P_{\sigma(N)}^{\alpha_{\sigma(N)}}+\ldots\right. \\
& +E_{(\sigma(a+n))}^{\gamma \gamma+1} P_{\sigma(1)}^{\alpha_{\sigma(1)}} \ldots P_{\sigma(a)}^{\gamma-1} P_{\sigma(a+1)}^{\gamma} \ldots P_{\sigma(a+n)}^{\gamma \rightarrow \gamma+1} \ldots P_{\sigma(a+l)=k}^{\gamma} \ldots P_{\sigma(N)}^{\alpha_{\sigma(N)}}+\ldots \\
& \left.+E_{(\sigma(a+1))}^{\gamma \gamma+1} P_{\sigma(1)}^{\alpha_{\sigma(1)}} \ldots P_{\sigma(a)}^{\gamma-1} P_{\sigma(a+1)}^{\gamma \rightarrow \gamma+1} \ldots P_{\sigma(a+l)=k}^{\gamma} \ldots P_{\sigma(N)}^{\alpha_{\sigma(N)}}\right] \\
& \times R_{\hat{\sigma}_{k} \sigma(N \ldots 1)}^{\bar{\sigma} \sigma^{-1} \hat{\sigma}_{k}^{-1} \sigma} \prod_{i=1}^{N} P_{\hat{\sigma}_{k} \sigma(i)}^{\alpha_{\hat{\sigma}_{k} \sigma(i)}} \prod_{i<j} \Delta_{i j}^{-1} \\
= & \sum_{k=1}^{N} E_{(k)}^{\gamma \gamma+1} \otimes_{j \neq k} G_{(j)}^{\gamma \gamma+1}(k, j) .
\end{aligned}
$$

Here in (IV.6) $\hat{\sigma}_{k}$ is the element of $\mathcal{S}_{N}$ which permutes the first abnormal sequence in the square bracket of (IV.6) to normal sequence and $G^{\gamma \gamma+1}(i, j)$ in (IV.7) has the following elements: For $1<\gamma+1 \leq m$,

$$
G^{\gamma \gamma+1}(i, j)_{k l}=\delta_{k, l}\left\{\begin{array}{cl}
2 & k=\gamma \\
\left(2 a_{i j}\right)^{-1} & k=\gamma+1 \\
1 & \text { otherwise }
\end{array}\right.
$$

for $\gamma=m$

$$
G^{\gamma+1}(i, j)_{k l}=\delta_{k, l}\left\{\begin{array}{cc}
2 & k=\gamma \\
1 & \text { otherwise }
\end{array},\right.
$$

and for $m+1 \leq \gamma<m+n$

$$
G^{\gamma \gamma+1}(i, j)_{k l}=\delta_{k, l}\left\{\begin{array}{cl}
\left(a_{i j}\right)^{-1} & k=\gamma \\
1 & \text { otherwise }
\end{array}\right.
$$

The non-simple generators are generated by the simple generators through the relation

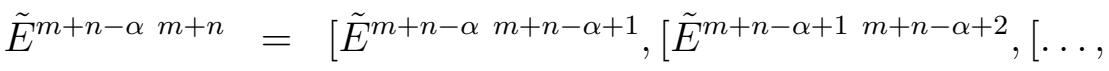

$$
\begin{aligned}
& \left.\left.\left.\left[\tilde{E}^{m+n-2} m+n-1, \tilde{E}^{m+n-1} m+n\right] \ldots\right]\right]\right] \text {. }
\end{aligned}
$$

We have:

1. For $m=0$, the $g l(m \mid n)$ supersymmetric model degenerates to the bosonic $g l(n)$ model, which has been discussed in [6] by Albert et al.

2. For $n=0, \alpha<m$,

$$
\tilde{E}^{m-\alpha m}=\sum_{k=1}^{\alpha} \sum_{i_{1} \neq \ldots \neq i_{k}} \prod_{\gamma=1}^{k-1} \frac{\eta}{z_{i_{\gamma}}-z_{i_{\gamma+1}}} \sum_{\alpha=\beta_{0}>\ldots>\beta_{k}=0} \otimes_{l=1}^{k} E_{\left(i_{l}\right)}^{m-\beta_{l-1} m-\beta_{l}}
$$




$$
\begin{gathered}
\otimes \operatorname{diag}(1, \ldots, 1,2, \underbrace{a_{i_{1} j}^{-1}, \ldots, a_{i_{1} j}^{-1}}_{\beta_{0}-\beta_{1}}, \ldots, \underbrace{a_{i_{k-1} j}^{-1}, \ldots, a_{i_{k-1} j}^{-1}}_{\beta_{k}-\beta_{k-1}}, \\
\underbrace{a_{i_{k} j}^{-1}, \ldots, a_{i_{k} j}^{-1}}_{\beta_{k-1}-\beta_{k}-1},\left(2 a_{i_{k} j}\right)^{-1})_{(j)},
\end{gathered}
$$

3. For $m, n \geq 1$ and $\alpha<n$,

$$
\begin{aligned}
\tilde{E}^{m+n-\alpha} m+n= & \sum_{k=1}^{\alpha} \sum_{i_{1} \neq \ldots \neq i_{k}} \prod_{\gamma=1}^{k-1} \frac{\eta}{z_{i_{\gamma+1}}-z_{i_{\gamma}}} \sum_{\alpha=\beta_{0}>\ldots>\beta_{k}=0} \otimes_{l=1}^{k} E_{\left(i_{l}\right)}^{m+n-\beta_{l-1}}{ }^{m+n-\beta_{l}} \\
& \otimes \operatorname{diag}(1, \ldots, 1, \underbrace{a_{i_{1} j}^{-1}, \ldots, a_{i_{1} j}^{-1}}_{\beta_{0}-\beta_{1}}, \ldots, \underbrace{a_{i_{k} j}^{-1}, \ldots, a_{i_{k} j}^{-1}}_{\beta_{k-1}-\beta_{k}}, 1)_{(j)}, \quad \text { IV } .
\end{aligned}
$$

4. For $m, n \geq 1$ and $\alpha \geq n$,

$$
\begin{aligned}
& \tilde{E}^{m+n-\alpha} m+n=\sum_{k=1}^{\alpha} \sum_{i_{1} \neq \ldots \neq i_{k}} \prod_{\gamma=1}^{\min (k-1, n-1)} \frac{\eta}{z_{i_{\gamma+1}}-z_{i_{\gamma}}} \prod_{\gamma=n+1}^{k-1} \frac{\eta}{z_{i_{\gamma}}-z_{i_{\gamma+1}}} \\
& \times \sum_{\alpha=\beta_{0}>\ldots>\beta_{k}=0} \otimes_{l=1}^{k} E_{\left(i_{l}\right)}^{m+n-\beta_{l-1} m+n-\beta_{l}} \\
& \otimes \operatorname{diag}(1, \ldots, 1,2, \underbrace{a_{i_{1} j}^{-1}, \ldots, a_{i_{1} j}^{-1}}_{\beta_{0}-\beta_{1}}, \ldots, \underbrace{a_{i_{p} j}^{-1}, \ldots, a_{i_{p} j}^{-1}}_{\beta_{p-1}-\beta_{p}} \text {, }
\end{aligned}
$$

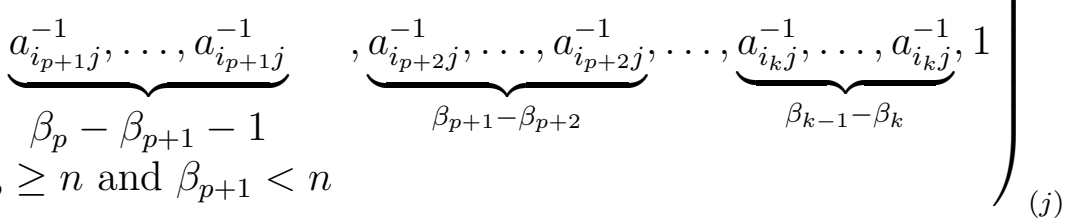

\section{IV.2 Elements of the monodromy matrix in the F-basis}

In the $F$-basis, the monodromy matrix $T(u)$, (II.9), becomes

$$
\tilde{T}(u)=\left(\begin{array}{cccc}
\tilde{A}_{11}(u) & \cdots & \tilde{A}_{1 m+n-1}(u) & \tilde{B}_{1}(u) \\
\vdots & \vdots & \vdots & \vdots \\
\tilde{A}_{m+n-11}(u) & \cdots & \tilde{A}_{m+n-1 m+n-1}(u) & \tilde{B}_{m+n-1}(u) \\
\tilde{C}_{1}(u) & \cdots & \tilde{C}_{m+n-1}(u) & \tilde{D}(u)
\end{array}\right)
$$


We first study the diagonal element $\tilde{D}(u)$. Acting the $F$-matrix on $D(u)$, we have

$$
\begin{aligned}
F_{1 \ldots N} D & =\sum_{\sigma \in \mathcal{S}_{N}} \sum_{\alpha_{\sigma(1) \ldots \alpha_{\sigma(N)}}}^{*} S\left(c, \sigma, \alpha_{\sigma}\right) \prod_{i=1}^{N} P_{\sigma(i)}^{\alpha_{\sigma}} R_{1 \ldots N}^{\sigma} P_{0}^{m+n} T_{0,1 \ldots N} P_{0}^{m+n} \\
& \left.=\sum_{\sigma \in \mathcal{S}_{N}} \sum_{\alpha_{\sigma(1)} \ldots \alpha_{\sigma(N)}}^{*} S\left(c, \sigma, \alpha_{\sigma}\right) \prod_{i=1}^{N} P_{\sigma(i)}^{\alpha_{\sigma}} P_{0}^{m+n} T_{0, \sigma(1 \ldots N)} P_{0}^{m+n} R_{1 \ldots N}^{\sigma} \text {. (I }\right)
\end{aligned}
$$

Following [6], we can split the sum $\sum^{*}$ according to the number of occurrences of the index $m+n$.

$$
\begin{aligned}
F_{1 \ldots N} T^{m+n} m+n= & \sum_{\sigma \in \mathcal{S}_{N}} \sum_{k=0}^{N} \sum_{\alpha_{\sigma(1)} \ldots \alpha_{\sigma(N)}}^{*} S\left(c, \sigma, \alpha_{\sigma}\right) \prod_{j=N-k+1}^{N} \delta_{\alpha_{\sigma(j)}, m+n} P_{\sigma(j)}^{\alpha_{\sigma(j)}} \\
& \times \prod_{j=1}^{N-k} P_{\sigma(j)}^{\alpha_{\sigma(j)}} P_{0}^{m+n} T_{0, \sigma(1 \ldots N)} P_{0}^{m+n} R_{1 \ldots N}^{\sigma}
\end{aligned}
$$

Consider the prefactor of $R_{1 \ldots N}^{\sigma}$. We have

$$
\begin{aligned}
& \prod_{j=1}^{N-k} P_{\sigma(j)}^{\alpha_{\sigma(j)}} \prod_{j=N-k+1}^{N} P_{\sigma(j)}^{m+n} P_{0}^{m+n} T_{0, \sigma(1 \ldots N)} P_{0}^{m+n} \\
= & \prod_{j=1}^{N-k} P_{\sigma(j)}^{\alpha_{\sigma(j)}} \prod_{j=N-k+1}^{N}\left(R_{0 \sigma(j)}\right)_{m+n}^{m+n} m+n \\
= & \prod_{j=1}^{m+n} P_{\sigma(j)}^{\alpha_{\sigma(j)}} P_{0}^{m+n} T_{0, \sigma(1 \ldots N-k)} P_{0, \sigma(1 \ldots N-k)}^{m+n} P_{0}^{m+n} \prod_{j=N-k+1}^{N} P_{\sigma(j)}^{m+n} P_{\sigma(j)}^{m+n} \\
= & \prod_{i=1}^{N-k}\left(R_{0 \sigma(i)}\right)_{m+n+k+1}^{m+n \alpha_{\sigma(i)}} \prod_{j=1}^{N-k} P_{\sigma(j)}^{\alpha_{\sigma(j)}} \prod_{j=N-k+1}^{N} P_{\sigma(j)}^{m+n} \\
= & \prod_{i=1}^{N-k} a_{0 \sigma(i)} \prod_{j=1}^{N-k} P_{\sigma(j)}^{\alpha_{\sigma(j)}} \prod_{j=N-k+1}^{N} P_{\sigma(j)}^{m+n},
\end{aligned}
$$

where $c_{0 i}=c\left(u, z_{i}\right), a_{0 i}=a\left(u, z_{i}\right)$. Substituting (IV.18) into (IV.17), we have

$$
F_{1 \ldots N} T^{m+n m+n}=\otimes_{i=1}^{N} \operatorname{diag}\left(a_{0 i}, \ldots, a_{0 i}, 1\right)_{(i)} F_{1 \ldots N}
$$

Therefore,

$$
\tilde{D}(u)=\otimes_{i=1}^{N} \operatorname{diag}\left(a_{0 i}, \ldots, a_{0 i}, 1\right)_{(i)} .
$$

The creation operators in the monodromy matrix can then be obtained as follows:

$$
\tilde{C}_{\alpha}(u)=\left[\tilde{E}^{\alpha m+n}, \tilde{D}(u)\right], \quad(1 \leq \alpha<m+n),
$$


which follows from the $g l(m \mid n)$ invariance of the $R$-matrix, i.e. in terms of the monodromy matrix,

$$
\left[\tilde{T}(u), \tilde{E}_{(0)}^{\alpha \beta}+\tilde{E}^{\alpha \beta}\right]=0 .
$$

Substituting $\tilde{E}^{\alpha m+n}, \tilde{E}^{m+n \alpha}$ and $\tilde{T}^{m+n m+n}$ into the above relations yields for $n=0$,

$$
\begin{gathered}
\tilde{C}_{m-\alpha}(u)=-\sum_{k=1}^{\alpha} \sum_{i_{1} \neq \ldots \neq i_{k}} b_{0 i_{k}} \prod_{\gamma=1}^{k-1} \frac{a_{0 i_{\gamma}} \eta}{z_{i_{\gamma}}-z_{i_{\gamma}+1}} \sum_{\alpha=\beta_{0}>\ldots>\beta_{k}=0} \otimes_{l=1}^{k} E_{\left(i_{l}\right)}^{m-\beta_{l-1} m-\beta_{l}} \\
\otimes \operatorname{diag}(a_{0 j}, \ldots, a_{0 j}, 2 a_{0 j}, \underbrace{a_{0 j} a_{i_{1} j}^{-1}, \ldots, a_{0 j} a_{i_{1} j}^{-1}}_{\beta_{0}-\beta_{1}}, \\
\ldots, \underbrace{a_{0 j} a_{i_{k} j}^{-1}, \ldots, a_{0 j} a_{i_{k} j}^{-1}}_{\beta_{k-1}-\beta_{k}-1}, c_{0 j}\left(2 a_{i_{k}, j}\right)^{-1})_{(j)}
\end{gathered}
$$

for $m, n \geq 1$ and $\alpha<n$,

$$
\begin{aligned}
\tilde{C}_{m+n-\alpha}(u)= & \sum_{k=1}^{\alpha} \sum_{i_{1} \neq \ldots \neq i_{k}} b_{0 i_{k}} \prod_{\gamma=1}^{k-1} \frac{a_{0 i_{\gamma}} \eta}{z_{i_{\gamma+1}}-z_{i_{\gamma}}} \sum_{\alpha=\beta_{0}>\ldots>\beta_{k}=0} \otimes_{l=1}^{k} E_{\left(i_{l}\right)}^{m+n-\beta_{l-1} m+n-\beta_{l}} \\
& \otimes \operatorname{diag}(a_{0 j}, \ldots, a_{0 j}, \underbrace{a_{0 j} a_{i_{1} j}^{-1}, \ldots, a_{0 j} a_{i_{1} j}^{-1}}_{\beta_{0}-\beta_{1}}, \ldots, \underbrace{a_{0 j} a_{i_{k} j}^{-1}, \ldots, a_{0 j} a_{i_{k} j}^{-1}}_{\beta_{k-1}-\beta_{k}}, 1)
\end{aligned}
$$

and for $m, n \geq 1$ and $\alpha \geq n$,

$$
\begin{aligned}
& \tilde{C}_{m+n-\alpha}(u)=\sum_{k=1}^{\alpha} \sum_{i_{1} \neq \ldots \neq i_{k}} b_{0 i_{k}} \prod_{\gamma=1}^{\min (k-1, n-1)} \frac{a_{0 i_{\gamma}} \eta}{z_{i_{\gamma+1}}-z_{i_{\gamma}}} \prod_{\gamma=n+1}^{k-1} \frac{a_{0 i_{\gamma}} \eta}{z_{i_{\gamma}}-z_{i_{\gamma+1}}} \\
& \times \sum_{\alpha=\beta_{0}>\ldots>\beta_{k}=0} \otimes_{l=1}^{k} E_{\left(i_{l}\right)}^{m+n-\beta_{l-1} m+n-\beta_{l}} \\
& \otimes \operatorname{diag}(a_{0 j}, \ldots, a_{0 j}, 2 a_{0 j}, \underbrace{a_{0 j} a_{i_{1} j}^{-1}, \ldots, a_{0 j} a_{i_{1 j}}^{-1}}_{\beta_{0}-\beta_{1}}, \ldots, \underbrace{a_{0 j} a_{i_{p j}}^{-1}, \ldots, a_{0 j} a_{i_{p j} j}^{-1}}_{\beta_{p-1}-\beta_{p}}, \\
& \underbrace{a_{0 j} a_{i_{p+1 j}}^{-1}, \ldots, a_{0 j} a_{i_{p+1 j}}^{-1}}_{\beta_{p}-\beta_{p+1}-1}, \underbrace{a_{0 j} a_{i_{p+2 j}}^{-1}, \ldots, a_{0 j} a_{i_{p+2} j}^{-1}}_{\beta_{p+1}-\beta_{p+2}}, \\
& \beta_{p} \geq n \text { and } \beta_{p+1}<n
\end{aligned}
$$




$$
\ldots, \underbrace{a_{0 j} a_{i_{k} j}^{-1}, \ldots, a_{0 j} a_{i_{k} j}^{-1}}_{\beta_{k-1}-\beta_{k}}, 1)_{(j)} .
$$

Here, $a_{0 j}$ and $b_{0 j}$ stand for $a\left(u, z_{j}\right)$ and $b\left(u, z_{j}\right)$, respectively.

\section{V $g l(2 \mid 2)$ Bethe vectors in the $F$-basis}

In this section, specializing to the superconductive $g l(2 \mid 2)$ electronic system, we will resolve its nested Bethe vectors in the $F$-basis.

In the framework of the algebraic Bethe ansatz, the pseudo-vacuum state is

$$
\mid 0>=\otimes_{k=1}^{N}\left(\begin{array}{l}
0 \\
0 \\
0 \\
1
\end{array}\right)_{(k)}
$$

The Bethe vector of the model is then defined by

$$
\Phi_{N}\left(v_{1}, \ldots, v_{n_{1}}\right)=\sum_{d_{1} \ldots d_{n_{1}}}\left(\phi_{n_{1}}^{(1)}\right)^{d_{1} \ldots d_{n_{1}}} C_{d_{1}}\left(v_{1}\right) \ldots C_{d_{n_{1}}}\left(v_{n_{1}}\right) \mid 0>
$$

where $d_{i}=1,2,3,\left(\phi_{n_{1}}^{(1)}\right)^{d_{1} \ldots d_{n_{1}}}$ is a function of the spectral parameter $v_{j}$. In the algebraic Bethe ansatz, $\left(\phi_{n_{1}}^{(1)}\right)^{d_{1} \ldots d_{n_{1}}}$ is also associated with the 3 -dimensional nested Bethe vector

$$
\phi_{n_{1}}^{(1)}\left(v_{1}^{(1)}, \ldots, v_{n_{2}}^{(1)}\right)=\sum_{d_{1} \ldots d_{n_{2}}}\left(\phi_{n_{2}}^{(2)}\right)^{d_{1} \ldots d_{n_{2}}} C_{d_{1}}^{(1)}\left(v_{1}^{(1)}\right) \ldots C_{d_{n_{2}}}^{(1)}\left(v_{n_{2}}^{(1)}\right) \mid 0>^{(1)}
$$

where $d_{i}=1,2, \mid 0>^{(1)}$ is the nested pseudo-vacuum state

$$
\mid 0>^{(1)}=\otimes_{k=1}^{n_{1}}\left(\begin{array}{l}
0 \\
0 \\
1
\end{array}\right)
$$

$\left(\phi_{n_{2}}^{(2)}\right)^{d_{1} \ldots d_{n_{2}}}$ is a function of the spectral parameter $v_{j}^{(1)}$, and $C^{(1)}$ 's are the creation operators of the nested $g l(2 \mid 1)$ system. Here $\phi_{n_{2}}^{(2)}$ is the second level nested Bethe vector associated with the $g l(2)$ model. As usual, the second level nested Bethe vector is defined by

$$
\phi_{n_{2}}^{(2)}\left(v_{1}^{(2)}, \ldots, v_{n_{3}}^{(2)}\right)=C^{(2)}\left(v_{1}^{(2)}\right) \ldots C^{(2)}\left(v_{n_{3}}^{(2)}\right) \mid 0>^{(2)}
$$

with

$$
\mid 0>^{(2)}=\otimes_{k=1}^{n_{2}}\left(\begin{array}{l}
0 \\
1
\end{array}\right)_{(k)}
$$


Applying the $g l(2 \mid 2) F$-matrix, i.e. $m=2, n=2$ in (III.4), to the pseudo-vacuum state (V.1), we find it is invariant. This is due to the fact that only terms whose roots all equal to 4 , in the definition expression of $F^{(1)}$, produce non-zero results. Therefore the Bethe vector (V.2) in the $F$-basis becomes

$$
\begin{aligned}
\tilde{\Phi}_{N}\left(v_{1}, \ldots, v_{n_{1}}\right) & \equiv F_{1 \ldots N} \Phi_{N}\left(v_{1}, \ldots, v_{n_{1}}\right) \\
& =\sum_{d_{1} \ldots d_{n_{1}}}\left(\phi_{n_{1}}^{(1)}\right)^{d_{1} \ldots d_{n_{1}}} \tilde{C}_{d_{1}}\left(v_{1}\right) \ldots \tilde{C}_{d_{n_{1}}}\left(v_{n_{1}}\right) \mid 0>.
\end{aligned}
$$

For the Bethe vector (V.7), one checks that the spectral parameters of the system preserve the exchange symmetry

$$
\tilde{\Phi}_{N}\left(v_{\sigma(1)}, \ldots, v_{\sigma\left(n_{1}\right)}\right)=\tilde{\Phi}_{N}\left(v_{1}, \ldots, v_{n_{1}}\right),
$$

while the creation operators satisfy the following commutation relation [14]

$$
\tilde{C}_{i}(u) \tilde{C}_{j}(v)=(-1)^{[i][j]} \frac{1}{a(v, u)} \tilde{C}_{j}(v) \tilde{C}_{i}(u)-(-1)^{[j]} \frac{b(v, u)}{a(v, u)} \tilde{C}_{j}(u) \tilde{C}_{i}(v) .
$$

These two properties mean that we may propose the following special sequence of the creation operators with $p_{1}$ number of $d_{i}=1, p_{2}-p_{1}$ number of $d_{i}=2$ and $n_{1}-p_{2}$ number of $d_{i}=3$ :

$$
\tilde{C}_{1}\left(v_{1}\right) \ldots \tilde{C}_{1}\left(v_{p_{1}}\right) \tilde{C}_{2}\left(v_{p_{1}+1}\right) \ldots \tilde{C}_{2}\left(v_{p_{2}}\right) \tilde{C}_{3}\left(v_{p_{2}+1}\right) \ldots \tilde{C}_{3}\left(v_{n_{1}}\right) .
$$

With the help of the commutation relation ( $(\underline{\nabla .9})$, we may rewrite the sequence as

$$
\begin{aligned}
& \tilde{C}_{1}\left(v_{1}\right) \ldots \tilde{C}_{1}\left(v_{p_{1}}\right) \tilde{C}_{2}\left(v_{p_{1}+1}\right) \ldots \tilde{C}_{2}\left(v_{p_{2}}\right) \tilde{C}_{3}\left(v_{p_{2}+1}\right) \ldots \tilde{C}_{3}\left(v_{n_{1}}\right) \\
= & h\left(v_{1}, \ldots, v_{n_{1}}\right) \tilde{C}_{3}\left(v_{p_{2}+1}\right) \ldots \tilde{C}_{3}\left(v_{n_{1}}\right) \tilde{C}_{2}\left(v_{p_{1}+1}\right) \ldots \tilde{C}_{2}\left(v_{p_{2}}\right) \\
& \times \tilde{C}_{1}\left(v_{1}\right) \ldots \tilde{C}_{1}\left(v_{p_{1}}\right)+\ldots
\end{aligned}
$$

with

$$
h\left(v_{1}, \ldots, v_{n_{1}}\right)=\prod_{k=1}^{p_{1}} \prod_{l=p_{1}+1}^{p_{2}}\left(-\frac{1}{a\left(v_{l}, v_{k}\right)}\right) \prod_{k=1}^{p_{2}} \prod_{l=p_{2}+1}^{n_{1}}\left(\frac{1}{a\left(v_{l}, v_{k}\right)}\right) .
$$

Here the prefactor $h$ comes from the first term of (V.9), and "..." stands for the other terms contributed by the second term. Considering the exchange symmetry (V.8), one easily checks that the other terms "..." can be represented by

$$
C_{3}\left(v_{\sigma\left(p_{2}+1\right)}\right) \ldots \tilde{C}_{3}\left(v_{\sigma\left(n_{1}\right)}\right) \tilde{C}_{2}\left(v_{\sigma\left(p_{1}+1\right)}\right) \ldots \tilde{C}_{2}\left(v_{\sigma\left(p_{2}\right)}\right) \tilde{C}_{1}\left(v_{\sigma(1)}\right) \ldots \tilde{C}_{1}\left(v_{\sigma\left(p_{1}\right)}\right),
$$

where $\sigma \in \mathcal{S}_{n_{1}}$. Substituting (V.10) into the Bethe vector (V.7), we then propose the following Bethe vector $\Phi_{N}^{\left(p_{1}, p_{2}\right)}$ corresponding to the quantum number $p_{1}$ and $p_{2}$ :

$$
\begin{aligned}
& \tilde{\Phi}_{N}^{\left(p_{1}, p_{2}\right)}\left(v_{1}, \ldots, v_{n_{1}}\right) \\
= & \left(\phi_{n_{1}}^{(1)}\right)^{1 \ldots 12 \cdots 23 \cdots 3} \tilde{C}_{1}\left(v_{1}\right) \ldots \tilde{C}_{1}\left(v_{p_{1}}\right) \tilde{C}_{2}\left(v_{p_{1}+1}\right) \ldots \tilde{C}_{2}\left(v_{p_{2}}\right) \tilde{C}_{3}\left(v_{p_{2}+1}\right) \ldots \tilde{C}_{3}\left(v_{n_{1}}\right) \mid 0> \\
= & \frac{1}{p_{1} !\left(p_{2}-p_{1}\right) !\left(n_{1}-p_{2}\right) !} \sum_{\sigma \in \mathcal{S}_{n_{1}}}\left(\phi_{n_{1}}^{(1), \sigma}\right)^{1 \cdots 12 \cdots 23 \cdots 3} \tilde{C}_{3}\left(v_{\sigma\left(p_{2}+1\right)}\right) \ldots \tilde{C}_{3}\left(v_{\sigma\left(n_{1}\right)}\right) \\
& \times \tilde{C}_{2}\left(v_{\sigma\left(p_{1}+1\right)}\right) \ldots \tilde{C}_{2}\left(v_{\sigma\left(p_{2}\right)}\right) \tilde{C}_{1}\left(v_{\sigma(1)}\right) \ldots \tilde{C}_{1}\left(v_{\sigma\left(p_{1}\right)}\right) \mid 0>
\end{aligned}
$$


where $\sigma$ in $\phi_{n_{1}}^{(1), \sigma}$ implies that the permutation of the inhomogeneous parameters of the original nested Bethe vector.

In view of (IV.24) and (IV.25), the $g l(2 \mid 2)$ creation operators $C_{i}(i=1,2,3)$ in the $F$-basis take the form

$$
\begin{aligned}
& \tilde{C}_{3}=\sum_{i=1}^{N} b_{0 i} E_{(i)}^{34} \otimes_{j \neq i} \operatorname{diag}\left(a_{0 j}, a_{0 j}, a_{0 j} a_{i j}^{-1}, 1\right)_{(j)}, \\
& \tilde{C}_{2}=\sum_{i=1}^{N} b_{0 i} E_{(i)}^{24} \otimes_{j \neq i} \operatorname{diag}\left(a_{0 j}, 2 a_{0 j}, a_{0 j} a_{i j}^{-1}, 1\right)_{(j)}+\ldots, \\
& \tilde{C}_{1}=\sum_{i=1}^{N} b_{0 i} E_{(i)}^{14} \otimes_{j \neq i} \operatorname{diag}\left(2 a_{0 j}, a_{0 j} a_{i j}^{-1}, a_{0 j} a_{i j}^{-1}, 1\right)_{(j)}+\ldots,
\end{aligned}
$$

where "..." stands for terms which contain more than one generators, e.g. $E_{i}^{12} \otimes E_{j}^{24}$ etc.. Applying $\tilde{C}_{2}$ and $\tilde{C}_{1}$ to the pseudo-vacuum state (V.1), one finds that all other terms equal to zero. Therefore, substituting (V.14) $-(\mathrm{V.16})$ into (V.13), we obtain

$$
\begin{aligned}
& \tilde{\Phi}_{N}^{\left(p_{1}, p_{2}\right)}\left(v_{1}, \ldots, v_{n_{1}}\right) \\
= & \frac{1}{p_{1} !\left(p_{2}-p_{1}\right) !\left(n-p_{2}\right) !} \sum_{i_{1}<\ldots<i_{p_{1}}} \sum_{i_{p_{1}+1}<\ldots<i_{p_{2}}} \\
& \times \sum_{i_{p_{2}+1}<\ldots<i_{n_{1}}} B_{n_{1},\left(p_{1}, p_{2}\right)}^{(0)}\left(v_{1}, \ldots, v_{n_{1}} ; v_{1}^{(1)}, \ldots, v_{p_{1}}^{(1)}, \ldots, v_{p_{2}}^{(1)} \mid z_{i_{1}}, \ldots, z_{i_{n_{1}}}\right) \\
& \times \prod_{j=p_{2+1}}^{n_{1}} E_{\left(i_{j}\right)}^{34} \prod_{j=p_{1+1}}^{p_{2}} E_{\left(i_{j}\right)}^{24} \prod_{j=i_{1}}^{p_{1}} E_{\left(i_{j}\right)}^{14} \mid 0>
\end{aligned}
$$

where $\left\{i_{k_{a}+1}, i_{k_{a}+1}, \ldots, i_{k_{a+1}}\right\} \cap\left\{i_{k_{b}+1}, i_{k_{b}+1}, \ldots, i_{k_{b+1}}\right\}=\varnothing\left(k_{a} \neq k_{b}\right.$ and $\left.k_{a}, k_{b} \in\left\{0, p_{1}, p_{2}\right\}\right)$ and

$$
\begin{aligned}
& B_{n_{1},\left(p_{1}, p_{2}\right)}^{(0)}\left(v_{1}, \ldots, v_{n_{1}} ; v_{1}^{(1)}, \ldots, v_{p_{1}}^{(1)}, \ldots, v_{p_{2}}^{(1)} \mid z_{i_{1}}, \ldots, z_{i_{n_{1}}}\right) \\
= & \sum_{\sigma \in S_{n}} \prod_{k=1}^{p_{1}} \prod_{l=p_{1}+1}^{p_{2}}\left(-\frac{a\left(v_{\sigma(l)}, z_{i_{k}}\right)}{a\left(v_{\sigma(l)}, v_{\sigma(k)}\right)}\right) \prod_{k=1}^{p_{2}} \prod_{l=p_{2}+1}^{n_{1}}\left(\frac{a\left(v_{\sigma(l)}, z_{i_{k}}\right)}{a\left(v_{\sigma(l)}, v_{\sigma(k)}\right)}\right) \\
& \times\left(\phi_{n_{1}}^{(1), \sigma}\right)^{1 \ldots 12 \cdots 23 \cdots 3} B_{n-p_{2}}\left(v_{\sigma\left(p_{2}+1\right)}, \ldots, v_{\sigma\left(n_{1}\right)} \mid z_{i_{p_{1}+1}}, \ldots, z_{i_{n_{1}}}\right) \\
& \times B_{p_{2}-p_{1}}^{*}\left(v_{\sigma\left(p_{1}+1\right)}, \ldots, v_{\sigma\left(p_{2}\right)} \mid z_{i_{p_{1}+1}}, \ldots, z_{i_{p_{2}}}\right) B_{p_{1}}^{*}\left(v_{\sigma(1)}, \ldots, v_{\sigma\left(p_{1}\right)} \mid z_{i_{1}}, \ldots, z_{i_{p_{1}}}\right)
\end{aligned}
$$

with

$$
\begin{aligned}
& B_{p}\left(v_{1}, \ldots, v_{p} \mid z_{1}, \ldots, z_{p}\right)=\sum_{\sigma \in \mathcal{S}_{p}} \prod_{m=1}^{p} b\left(v_{m}, z_{\sigma(m)}\right) \prod_{l=m+1}^{p} \frac{a\left(v_{m}, z_{\sigma(l)}\right)}{a\left(z_{\sigma(m)}, z_{\sigma(l)}\right)} \\
& B_{p}^{*}\left(v_{1}, \ldots, v_{p} \mid z_{1}, \ldots, z_{p}\right)=\sum_{\sigma \in \mathcal{S}_{p}} \operatorname{sign}(\sigma) \prod_{m=1}^{p} b\left(v_{m}, z_{\sigma(m)}\right) \prod_{l=m+1}^{p} 2 a\left(v_{m}, z_{\sigma(l)}\right) .
\end{aligned}
$$


In (V.18), we still need to determine the form of $\left(\phi_{n_{1}}^{(1)}\right)^{1 \cdots 12 \cdots 23 \cdots 3}$, which should be evaluated in the original basis. Define $\tilde{\phi}_{n_{1}}^{(1)} \equiv F_{1 \ldots n_{1}}^{(1)} \phi_{n_{1}}^{(1)}$. we now examine the relation between $\phi_{n_{1}}^{(1)}$ and $\tilde{\phi}_{n_{1}}^{(1)}$.

Write the nested pseudo-vacuum vector (V.4) as

$$
\left|0>^{(1)} \equiv\right| 3 \cdots 3>^{(1)} \text {, }
$$

where the number of 3 is $n_{1}$. Then the nested Bethe vector (V.3) can be rewritten as

$$
\phi_{n_{1}}^{(1)}\left(v_{1}^{(1)} \ldots v_{p_{2}}^{(1)}\right) \equiv\left|\phi_{n_{1}}^{(1)}>=\sum_{d_{1} \ldots d_{n_{1}}}\left(\phi_{n_{1}}^{(1)}\right)^{d_{1} \ldots d_{n_{1}}}\right| d_{1} \ldots d_{n_{1}}>^{(1)} .
$$

Acting the $g l(2 \mid 1) F$-matrix $F^{(1)}$ from the left on the above equation, we have

$$
\tilde{\phi}_{n_{1}}^{(1)}\left(v_{1}^{(1)} \ldots v_{p_{2}}^{(1)}\right) \equiv\left|\tilde{\phi}_{n_{1}}^{(1)}>=F^{(1)}\right| \phi_{n_{1}}^{(1)}>=\sum_{d_{1} \ldots d_{n_{1}}}\left(\tilde{\phi}_{n_{1}}^{(1)}\right)^{d_{1} \ldots d_{n_{1}}} \mid d_{1} \ldots d_{n_{1}}>^{(1)} .
$$

It follows that

$$
\begin{aligned}
\left(\tilde{\phi}_{n_{1}}^{(1)}\right)^{1 \ldots 12 \ldots 23 \ldots 3}= & <1 \ldots 12 \ldots 23 \ldots 3\left|\tilde{\phi}_{n_{1}}^{(1)}>=<1 \ldots 12 \ldots 23 \ldots 3\right| F^{(1)} \mid \phi_{n_{1}}^{(1)}> \\
= & <1 \ldots 12 \ldots 23 \ldots 3 \mid \sum_{\sigma \in \mathcal{S}_{n_{2}}} \sum_{\alpha_{\sigma(1)} \ldots \alpha_{\sigma\left(n_{1}\right)}}^{*} \prod_{j=1}^{n_{1}} P_{\sigma(j)}^{\alpha_{\sigma(j)}} \\
& \times S\left(c, \sigma, \alpha_{\sigma}\right) R_{1 \ldots n_{1}}^{\sigma} \mid \phi_{n_{1}}^{(1)}> \\
= & <1 \ldots 12 \ldots 23 \ldots 3\left|\left\{\sum_{\alpha_{\sigma(1)} \ldots \alpha_{\sigma\left(n_{1}\right)}}^{*} \prod_{j=1}^{n_{1}} P_{\sigma(j)}^{\alpha_{\sigma(j)}}\right\}\right|_{\sigma=i d} \\
& \times S\left(c, \sigma, \alpha_{\sigma}\right) \mid \phi_{n_{1}}^{(1)}> \\
= & t(c)<1 \ldots 12 \ldots 23 \ldots 3 \mid \phi_{n_{1}}^{(1)}>=t(c)\left(\phi_{n_{1}}^{(1)}\right)^{1 \ldots 12 \ldots 23 \ldots 3}
\end{aligned}
$$

with $t(c)=\prod_{j>i=1}^{p_{1}}\left(1+c_{i j}\right) \prod_{j>i=p_{1}+1}^{p_{2}}\left(1+c_{i j}\right)$. It follows that $\left(\phi_{n_{1}}^{(1)}\right)^{1 \ldots 12 \ldots 23 \ldots 3}$ may be computed by the $F$-transformed version $\left(\tilde{\phi}_{n_{1}}^{(1)}\right)^{1 \ldots 12 \ldots 23 \ldots 3}$.

In the following, we compute $\left(\phi_{n_{1}}^{(1)}\right)^{1 \ldots 12 \cdots 23 \cdots 3}$ with the help of the nested $g l(2 \mid 1)$ $F$-basis.

1. The first level nested $g l(2 \mid 1)$ Bethe vector

Denoted by $F_{1 \ldots n_{1}}^{(1)}$ the $n_{1}$-site $g l(2 \mid 1) F$-matrix. Acting the $F$-matrix on (․4 $)$, one finds that the nested $g l(2 \mid 1)$ pseudo-vacuum state is also invariant. Thus in the $F$-basis, the nested Bethe vector (V.3) becomes

$$
\tilde{\phi}_{n_{1}}^{(1)}\left(v_{1}^{(1)}, \ldots, v_{n_{2}}^{(1)}\right)=\sum_{d_{1} \ldots d_{n_{2}}}\left(\phi_{n_{2}}^{(2)}\right)^{d_{1} \ldots d_{n_{2}}} \tilde{C}_{d_{1}}^{(1)}\left(v_{1}^{(1)}\right) \ldots \tilde{C}_{d_{n_{2}}}^{(1)}\left(v_{d_{n_{2}}}^{(1)}\right) \mid 0>^{(1)} .
$$

We may prove that the nested Bethe vector satisfies the following exchange symmetry

$$
\tilde{\phi}_{n_{1}}^{(1)}\left(v_{\sigma(1)}^{(1)}, \ldots, v_{\sigma\left(n_{2}\right)}^{(1)}\right)=\frac{1}{c_{1 \ldots n_{2}}^{\sigma}} \tilde{\phi}_{n_{1}}^{(1)}\left(v_{1}^{(1)}, \ldots, v_{n_{2}}^{(1)}\right)
$$


where $c_{1, \ldots, n}^{\sigma}$ has the decomposition law

$$
c_{1 \ldots n}^{\sigma^{\prime} \sigma}=c_{\sigma^{\prime}(1 \ldots n)}^{\sigma} c_{1 \ldots n}^{\sigma^{\prime}}
$$

with $c_{1 \ldots n}^{\sigma_{i}}=c_{i+1} \equiv c\left(v_{i}, v_{i+1}\right)$ for an elementary permutation $\sigma_{i}$.

This enable one to concentrate on a particularly simple term in the sum (V.24) of the form with $p_{1}$ number of $d_{i}=1$ and $n-p_{1}$ number of $d_{j}=2$ :

$$
\tilde{C}_{1}^{(1)}\left(v_{1}^{(1)}\right) \ldots \tilde{C}_{1}^{(1)}\left(v_{p_{1}}^{(1)}\right) \tilde{C}_{2}^{(1)}\left(v_{p_{1}+1}^{(1)}\right) \ldots \tilde{C}_{2}^{(1)}\left(v_{n_{2}}^{(1)}\right) .
$$

The commutation relation between $C_{i}(v)$ and $C_{j}(u)$ [14] in the $F$-basis becomes

$$
\tilde{C}_{i}^{(1)}(v) \tilde{C}_{j}^{(1)}(u)=-\frac{1}{a(u, v)} \tilde{C}_{j}^{(1)}(u) \tilde{C}_{i}^{(1)}(v)+\frac{b(u, v)}{a(u, v)} \tilde{C}_{j}^{(1)}(v) \tilde{C}_{i}^{(1)}(u) .
$$

Then using (V.28), all $\tilde{C}_{1}^{(1)}$ 's in (V.27) can be moved to the right of all $\tilde{C}_{2}^{(1)}$ 's, yielding

$$
\begin{aligned}
& \tilde{C}_{1}^{(1)}\left(v_{1}^{(1)}\right) \ldots \tilde{C}_{1}^{(1)}\left(v_{p_{1}}^{(1)}\right) \tilde{C}_{2}^{(1)}\left(v_{p_{1}+1}^{(1)} \ldots \tilde{C}_{2}^{(1)}\left(v_{n_{2}}^{(1)}\right)=\right. \\
= & g\left(v_{1}^{(1)}, \ldots, v_{n_{2}}^{(1)}\right) \tilde{C}_{2}^{(1)}\left(v_{p_{1}+1}^{(1)}\right) \ldots \tilde{C}_{2}^{(1)}\left(v_{n_{2}}^{(1)}\right) \tilde{C}_{1}^{(1)}\left(v_{1}^{(1)}\right) \ldots \tilde{C}_{1}^{(1)}\left(v_{p_{1}}^{(1)}\right)+\ldots,
\end{aligned}
$$

where $g\left(v_{1}^{(1)}, \ldots, v_{n_{2}}^{(1)}\right)=\prod_{k=1}^{p_{1}} \prod_{l=p_{1}+1}^{n_{2}}\left(-1 / a\left(v_{l}^{(1)}, v_{k}^{(1)}\right)\right)$ is the contribution from the first term of (V.28). As before, the other terms "..." can be given with the help of the permutation operator $\sigma \in \mathcal{S}_{n_{2}}$. Thus, we rewrite the nested Bethe vector as

$$
\begin{aligned}
& \tilde{\phi}_{n_{1}}^{(1), p_{1}}\left(v_{1}^{(1)}, \ldots, v_{n_{2}}^{(1)}\right)= \\
= & \frac{1}{p_{1} !\left(n_{2}-p_{1}\right) !} \sum_{\sigma \in \mathcal{S}_{n_{2}}} c_{1 \ldots n_{2}}^{\sigma}\left(\phi_{n_{2}}^{(2), \sigma}\right)^{11 \ldots 12 \ldots 2} \prod_{k=1}^{p_{1}} \prod_{l=p_{1}+1}^{n_{2}}\left(-\frac{1}{a\left(v_{\sigma(l)}^{(1)}, v_{\sigma(k)}^{(1)}\right)}\right) \\
& \times \tilde{C}_{2}^{(1)}\left(v_{\sigma\left(p_{1}+1\right)}^{(1)}\right) \ldots \tilde{C}_{2}^{(1)}\left(v_{\sigma\left(n_{2}\right)}^{(1)}\right) \tilde{C}_{1}^{(1)}\left(v_{\sigma(1)}^{(1)}\right) \ldots \tilde{C}_{1}^{(1)}\left(v_{\sigma\left(p_{1}\right)}^{(1)}\right) \mid 0>.
\end{aligned}
$$

From (IV.24) and (IV.25), the $g l(2 \mid 1)$ creation operators in the $F$-basis are given by

$$
\begin{aligned}
& \tilde{C}_{2}=\sum_{i=1}^{N} b_{0 i} E_{(i)}^{23} \otimes_{j \neq i} \operatorname{diag}\left(a_{0 j}, 2 a_{0 j}, 1\right)_{(j)} \\
& \tilde{C}_{1}=\sum_{i=1}^{N} b_{0 i} E_{(i)}^{13} \otimes_{j \neq i} \operatorname{diag}\left(2 a_{0 j}, a_{0 j} a_{i j}^{-1}, 1\right)_{(j)}+\ldots
\end{aligned}
$$

Substituting these expressions of $\tilde{C}_{i}$ into (V.30), we have

$$
\begin{aligned}
& \tilde{\phi}_{n_{1}}^{(1), p_{1}}\left(v_{1}^{(1)}, \ldots, v_{n_{2}}^{(1)}\right) \\
= & \frac{1}{p_{1} !\left(n_{2}-p_{1}\right) !} \sum_{i_{1}<\ldots<i_{p_{1}}} \sum_{i_{p_{1}+1}<\ldots<i_{n_{2}}} B_{n_{2}, p_{1}}^{(1)}\left(v_{1}^{(1)}, \ldots, v_{n_{2}}^{(1)} ; v_{1}^{(2)}, \ldots, v_{p_{1}}^{(2)} \mid v_{i_{1}}, \ldots, v_{i_{n_{2}}}\right) \\
& \times \prod_{j=p_{1}+1}^{n_{2}} E_{\left(i_{j}\right)}^{23} \prod_{j=1}^{p_{1}} E_{\left(i_{j}\right)}^{13} \mid 0>
\end{aligned}
$$


where $\left\{i_{1}, i_{2}, \ldots, i_{p_{1}}\right\} \cap\left\{i_{p_{1}+1}, i_{p_{1}+2}, \ldots, i_{n_{2}}\right\}=\varnothing$ and

$$
\begin{aligned}
& B_{n_{2}, p_{1}}^{(1)}\left(v_{1}^{(1)}, \ldots, v_{n_{2}}^{(1)} ; v_{1}^{(2)}, \ldots, v_{p_{1}}^{(2)} \mid v_{i_{1}}, \ldots, v_{i_{n_{2}}}\right)= \\
= & \sum_{\sigma \in S_{n_{2}}} c_{1 \ldots n_{2}}^{\sigma} \prod_{k=1}^{p_{1}} \prod_{l=p_{1}+1}^{n_{2}}\left(-\frac{a\left(v_{\sigma(l)}^{(1)}, v_{i_{k}}\right)}{a\left(v_{\sigma(l)}^{(1)}-v_{\sigma(k)}^{(1)}\right)}\right) \\
& \times\left(\phi_{n_{2}}^{(2), \sigma}\right)^{11 \ldots 12 \ldots 2} B_{n_{2}-p_{1}}^{*}\left(v_{\sigma\left(p_{1}+1\right)}^{(1)}, \ldots, v_{\sigma\left(n_{2}\right)}^{(1)} \mid v_{i_{p_{1}+1}}, \ldots, v_{i_{n_{2}}}\right) \\
& \times B_{p_{1}}^{*}\left(v_{\sigma(1)}^{(1)}, \ldots, v_{\sigma\left(p_{1}\right)}^{(1)} \mid v_{i_{1}}, \ldots, v_{i_{p_{1}}}\right) .
\end{aligned}
$$

Denote by $F^{(2)}$ the second level nested $g l(2) F$-matrix. Applying the $F$-matrix to $\phi_{n_{2}}^{(2)}$, one obtains

$$
\left(\phi_{n_{2}}^{(2)}\right)^{11 \ldots 12 \ldots 2}=\left(t^{\prime}(c)\right)^{-1}\left(\tilde{\phi}_{n_{2}}^{(2)}\right)^{11 \ldots 12 \ldots 2}
$$

with $t^{\prime}(c)=\prod_{j>i=1}^{p_{1}}\left(1+c_{i j}\right) \prod_{j>i=p_{1}+1}^{n_{2}}\left(1+c_{i j}\right)$. Therefore as before, the $\left(\phi_{n_{2}}^{(2), \sigma}\right)^{11 \ldots 12 \ldots 2}$ in (V.34) can be determined in the $g l(2) F$-basis.

2. The second level nested $g l(2)$ Bethe vector

The $m=2$, and $n=0$ limit of (III.4) gives the $g l(2) F$-matrix $F^{(2)}$. In this $F$-basis, the $n_{2}$-site simple generator $E^{12}$ is given by

$$
\tilde{E}^{(12)}=\sum_{i=1}^{n_{2}} E_{(i)}^{12} \otimes_{j \neq i} \operatorname{diag}\left(2,\left(2 a_{i j}\right)^{-1}\right)_{(j)},
$$

the diagonal element $D^{(2)}(u)$ of the $g l(2)$ monodromy matrix is

$$
\tilde{D}^{(2)}(u)=\otimes_{i=1}^{n_{2}} \operatorname{diag}\left(a_{0 i}, c_{0 i}\right)_{(0)}
$$

and the creation operator $C^{(2)}(u)$ becomes

$$
\tilde{C}^{(2)}(u)=-\sum_{i=1}^{n_{2}} b_{0 i} E_{(i)}^{12} \otimes_{j \neq i} \operatorname{diag}\left(2 a_{0 j},\left(2 a_{i j}\right)^{-1} c_{0 j}\right)_{(j)} .
$$

Applying $F^{(2)}$ to the nested Bethe vector $\phi_{n_{2}}^{(2)}$, we obtain

$$
\begin{aligned}
\tilde{\phi}_{n_{2}}^{(2)}\left(v_{1}^{(2)}, \ldots, v_{n_{3}}^{(2)}\right) & \equiv F_{1 \ldots n_{2}}^{(1)} \phi^{(2)}\left(v_{1}^{(2)}, \ldots, v_{n_{3}}^{(2)}\right) \\
& =s_{n_{3}}(c) \tilde{C}^{(2)}\left(v_{1}^{(2)}\right) \tilde{C}^{(2)}\left(v_{2}^{(2)}\right) \ldots \tilde{C}^{(2)}\left(v_{n_{3}}^{(2)}\right) \mid 0>^{(2)}
\end{aligned}
$$

where $s_{n}(c)=\prod_{j>i=1}^{n}\left(1+c_{i j}\right)$ is from the action of $F^{(2)}$ on the nested pseudo-vacuum state $\mid 0>^{(1)}$. With the help of $(\overline{V .38})$, the $F$-transformed nested Bethe vector is given by

$$
\begin{aligned}
& \tilde{\phi}_{n_{2}}^{(2)}\left(v_{1}^{(2)}, \ldots, v_{n_{3}}^{(2)}\right)=s_{n_{3}}(c) \tilde{C}^{(2)}\left(v_{1}^{(2)}\right) \ldots \tilde{C}^{(2)}\left(v_{n_{3}}^{(2)}\right) \mid 0>>^{(2)} \\
= & s_{n_{3}}(c) \sum_{i_{1}<\ldots<i_{n_{3}}} B_{n_{3}}^{(2)}\left(v_{1}^{(2)}, \ldots, v_{n_{3}}^{(2)} \mid v_{i_{1}}^{(1)}, \ldots, v_{i_{n_{3}}}^{(1)}\right) E_{\left(i_{1}\right)}^{12} \ldots E_{\left(i_{n_{3}}\right)}^{12} \mid 0>^{(2)}
\end{aligned}
$$


where

$$
\begin{aligned}
& B_{n_{3}}^{(2)}\left(v_{1}^{(2)}, \ldots, v_{n_{3}}^{(2)} \mid v_{2}^{(1)}, \ldots, v_{n_{3}}^{(1)}\right) \\
= & \sum_{\sigma \in \mathcal{S}_{n}} \prod_{k=1}^{n_{3}}\left(-b\left(v_{k}^{(2)}, v_{\sigma(k)}^{(1)}\right) \prod_{j \neq \sigma(1), \ldots, \sigma(k)}^{n_{2}} \frac{c\left(v_{k}^{(2)}, v_{j}^{(1)}\right)}{2 a\left(v_{\sigma(k)}^{(1)}, v_{j}^{(1)}\right)} \prod_{l=k+1}^{m} 2 a\left(v_{k}^{(2)}, v_{\sigma(l)}^{(1)}\right) .\right.
\end{aligned}
$$

Here we note that if we exchange the spectral parameter in (V.39), the $g l(2)$ Bethe vector is invariant. Therefore substituting the representation of $\tilde{\phi}^{(2)}$, i.e. $B^{(2)}$, into (V.34), we may rewrite the nested $g l(2 \mid 1)$ Bethe vector as

$$
\begin{aligned}
& \tilde{\phi}_{n_{1}, p_{1}}^{(1)}\left(v_{1}^{(1)}, \ldots, v_{n_{2}}^{(1)}\right) \\
= & \frac{s_{p_{1}}(c)}{p_{1} !\left(n_{2}-p_{1}\right) !} \sum_{i_{1}<\ldots<i_{p_{1}}} \sum_{i_{p_{1}+1}<\ldots<i_{n_{2}}} B_{n_{2}, p_{1}}^{(1)}\left(v_{1}^{(1)}, \ldots, v_{n_{2}}^{(1)} ; v_{1}^{(2)}, \ldots, v_{p_{1}}^{(2)} \mid v_{i_{1}}, \ldots, v_{i_{n_{2}}}\right) \\
& \times \prod_{j=p_{1}+1}^{n_{2}} E_{\left(i_{j}\right)}^{23} \prod_{j=1}^{p_{1}} E_{\left(i_{j}\right)}^{13} \mid 0>
\end{aligned}
$$

with

$$
\begin{aligned}
& B_{n_{2}, p_{1}}^{(1)}\left(v_{1}^{(1)}, \ldots, v_{n_{2}}^{(1)} ; v_{1}^{(2)}, \ldots, v_{p_{1}}^{(2)} \mid v_{i_{1}}, \ldots, v_{i_{n_{2}}}\right) \\
= & \sum_{\sigma \in S_{n_{2}}} c_{1 \ldots n_{2}}^{\sigma}\left(\prod_{\epsilon=0}^{1} \prod_{\sigma(j)>\sigma(i)=p_{\epsilon}+1}^{p_{\epsilon+1}}\left(1+c_{\sigma(i) \sigma(j)}\right)\right)^{-1} \\
& \times \prod_{k=1}^{p_{1}} \prod_{l=p_{1}+1}^{n_{2}}\left(-\frac{a\left(v_{\sigma(l)}^{(1)}, v_{i_{k}}\right)}{a\left(v_{\sigma(l)}^{(1)}, v_{\sigma(k)}^{(1)}\right)}\right) \\
& \times B_{p_{1}}^{(2)}\left(v_{1}^{(2)}, \ldots, v_{p_{1}}^{(2)} \mid v_{\sigma(1)}^{(1)}, \ldots, v_{\sigma\left(p_{1}\right)}^{(1)}\right) \\
& \times B_{n_{2}-p_{1}}^{*}\left(v_{\sigma\left(p_{1}+1\right)}^{(1)}, \ldots, v_{\sigma\left(n_{2}\right)}^{(1)} \mid v_{i_{p_{1}+1}}, \ldots, v_{i_{n_{2}}}\right) \\
& \times B_{p_{1}}^{*}\left(v_{\sigma(1)}^{(1)}, \ldots, v_{\sigma\left(p_{1}\right)}^{(1)} \mid v_{i_{1}}, \ldots, v_{i_{p_{1}}}\right) .
\end{aligned}
$$

Having resolved the nested $g l(2 \mid 1)$ Bethe vector, we now go back the Bethe vectors of $g l(2 \mid 2)$ electronic model. By the exchange symmetry of the $g l(2 \mid 1)$ Bethe vector $(\mathbb{V . 2 5})$, we represent the Bethe vector (V.44) in the F-basis by

$$
\begin{aligned}
& \tilde{\Phi}_{N}^{\left(p_{1}, p_{2}\right)}\left(v_{1}, \ldots, v_{n_{1}}\right) \\
= & \frac{s_{p_{1}}(c)}{\left(p_{1} !\right)^{2}\left(\left(p_{2}-p_{1}\right) !\right)^{2}\left(n_{1}-p_{2}\right) !} \sum_{i_{1}<\ldots<i_{p_{1}}} \sum_{i_{p_{1}+1}<\ldots<i_{p_{2}}} \\
& \times \sum_{i_{p_{2}+1}<\ldots<i_{n_{1}}} B_{n_{1},\left(p_{1}, p_{2}\right)}^{(0)}\left(v_{1}, \ldots, v_{n_{1}} ; v_{1}^{(1)}, \ldots, v_{p_{1}}^{(1)}, \ldots, v_{p_{2}}^{(1)} \mid z_{i_{1}}, \ldots, z_{i_{n_{1}}}\right)
\end{aligned}
$$




$$
\times \prod_{j=p_{2}+1}^{n_{1}} E_{\left(i_{j}\right)}^{34} \prod_{j=p_{1}+1}^{p_{2}} E_{\left(i_{j}\right)}^{24} \prod_{j=1}^{p_{1}} E_{\left(i_{j}\right)}^{14} \mid 0>
$$

with

$$
\begin{aligned}
& B_{n_{1},\left(p_{1}, p_{2}\right)}^{(0)}\left(v_{1}, \ldots, v_{n_{1}} ; v_{1}^{(1)}, \ldots, v_{p_{2}}^{(1)} \mid z_{i_{1}}, \ldots, z_{i_{n_{1}}}\right) \\
= & \sum_{\sigma \in S_{n_{1}}}\left(\prod_{\epsilon=0}^{1} \prod_{\sigma(j)>\sigma(i)=p_{\epsilon}+1}^{p_{\epsilon+1}}\left(1+c_{\sigma(i) \sigma(j)}\right)\right)^{-1} \\
& \times \prod_{k=1}^{p_{1}} \prod_{l=p_{1}+1}^{p_{2}}\left(-\frac{a\left(v_{\sigma(l)}, z_{i_{k}}\right)}{a\left(v_{\sigma(l)}, v_{\sigma(k)}\right)}\right) \prod_{k=1}^{p_{2}} \prod_{l=p_{2}+1}^{n_{1}} \frac{a\left(v_{\sigma(l)}, z_{i_{k}}\right)}{a\left(v_{\sigma(l)}, v_{\sigma(k)}\right)} \\
& \times B_{p_{2}, p_{1}}^{(1)}\left(v_{1}^{(1)}, \ldots, v_{p_{2}}^{(1)} ; v_{1}^{(2)}, \ldots, v_{p_{1}}^{(2)} \mid v_{\sigma\left(i_{1}\right)}, \ldots, v_{\sigma\left(i_{p_{2}}\right)}\right) \\
& \times B_{n-p_{2}}\left(v_{\sigma\left(p_{2}+1\right)}, \ldots, v_{\sigma\left(n_{1}\right)} \mid z_{i_{p_{1}+1}}, \ldots, z_{i_{n_{1}}}\right) \\
& \times B_{p_{2}-p_{1}}^{*}\left(v_{\sigma\left(p_{1}+1\right)}, \ldots, v_{\sigma\left(p_{2}\right)} \mid z_{i_{p_{1}+1}}, \ldots, z_{i_{p_{2}}}\right) \\
& \times B_{p_{1}}^{*}\left(v_{\sigma(1)}, \ldots, v_{\sigma\left(p_{1}\right)} \mid z_{i_{1}}, \ldots, z_{i_{p_{1}}}\right) .
\end{aligned}
$$

\section{The resolution of the $g l(m \mid n)$ nested Bethe vec- tors in the $F$-basis}

In this section, we generalize the results in the previous section to the $g l(m \mid n)$ supersymmetric model. The procedure is similar to that of the $g l(2 \mid 2)$ case. Here we only give the final results.

Associated with the $(m+n)$-dimensional $g l(m \mid n)$ representation space, we have the orthogonal states $\mid j>(j=1,2, \ldots, m+n)$ defined by

$$
\left|1>=\left(\begin{array}{c}
1 \\
0 \\
\vdots \\
0
\end{array}\right) \quad, \ldots, \quad\right| m+n>=\left(\begin{array}{c}
0 \\
0 \\
\vdots \\
1
\end{array}\right)
$$

Consider the $g l(m \mid n)$ Bethe state vectors with quantum numbers $p_{1}, p_{2}-p_{1}, \ldots$, $p_{m+n-2}-p_{m+n-3}, n_{1}-p_{m+n-2}$, and $N-n_{1}$, which label the numbers of state $\mid 1>$, $|2>, \ldots| m+n>$, in the Bethe state, respectively. Define the pseudo-vacuum state of the $N$-site system

$$
\left|0>=\otimes_{k=1}^{N}\right| m+n>_{(k)} .
$$

Then the $g l(m \mid n)$ Bethe vector in the $F$-basis corresponding to the special quantum numbers, $\Phi_{N}^{\left(p_{1}, \ldots, p_{m+n-2}\right)}\left(v_{1}, \ldots, v_{n_{1}}\right)$, is given by

$$
\tilde{\Phi}_{N}^{\left(p_{1}, \ldots, p_{m+n-2}\right)}\left(v_{1}, \ldots, v_{n_{1}}\right)
$$




$$
\begin{aligned}
& =\frac{\prod_{\alpha=1}^{m-1} s_{p_{\alpha}}(c) p_{1} !}{\prod_{\alpha=1}^{m+n-1}\left(\left(p_{\alpha}-p_{\alpha-1}\right) !\right)^{m+n-\alpha}} \sum_{i_{1}<\ldots<i_{p_{1}}} \sum_{i_{p_{1}+1}<\ldots<i_{p_{2}}} \ldots \sum_{i_{p_{m+2}-2}+1<\ldots<i_{n_{1}}} \\
& \times B_{n_{1},\left(p_{1}, \ldots, p_{m+n-2}\right)}^{(0)}\left(v_{1}, \ldots, v_{n_{1}} ; v_{1}^{(1)}, \ldots, v_{p_{1}}^{(1)}, \ldots, v_{p_{m+n-2}}^{(1)} \mid z_{i_{1}}, \ldots, z_{i_{n_{1}}}\right) \\
& \times \prod_{\alpha=1}^{m+n-1} \prod_{j=p_{m+n-\alpha-1}+1}^{p_{m+n-\alpha}} E_{\left(i_{j}\right)}^{m+n-\alpha} m \mid 0>
\end{aligned}
$$

where $\left\{i_{k_{a}+1}, i_{k_{a}+2}, \ldots, i_{k_{a+1}}\right\} \cap\left\{i_{k_{b}+1}, i_{k_{b}+2}, \ldots, i_{k_{b+1}}\right\}=\varnothing \quad\left(k_{a} \neq k_{b}\right.$ and $k_{a}, k_{b} \in$ $\left.\left\{p_{0}, p_{1} \ldots, p_{m+n-2}\right\}\right)$, for $m=1$,

$$
\begin{aligned}
& B_{n_{1},\left(p_{1}, \ldots, p_{m+n-2}\right)}^{(0)}\left(v_{1}, \ldots, v_{n_{1}} ; v_{1}^{(1)}, \ldots, v_{p_{1}}^{(1)}, \ldots, v_{p_{m+n-2}}^{(1)} \mid z_{i_{1}}, \ldots, z_{i_{n_{1}}}\right) \\
= & B_{p_{1}}^{*}\left(v_{1}^{(n-1)}, \ldots, v_{p_{1}}^{(n-1)} \mid v_{\sigma^{2}(1)}^{(n-2)}, \ldots, v_{\sigma^{2}\left(p_{1}\right)}^{(n-2)}\right) \\
\times & \prod_{\beta=2}^{n}\left[\sum_{\sigma^{\beta} \in \mathcal{S}_{p_{\beta}}}\left(\prod_{\sigma^{\beta}(j)>\sigma^{\beta}(i)=1}^{p_{1}}\left(1+c_{\sigma^{\beta}(i) \sigma^{\beta}(j)}\right)\right) \prod_{\alpha=1}^{n-1} \prod_{k=1}^{p_{\alpha}} \prod_{l=p_{\alpha}+1}^{p_{\alpha+1}} \frac{a\left(v_{\sigma^{\beta}(l)}^{(n-\beta)}, v_{\sigma^{\beta+1}\left(k_{\alpha}\right)}^{(n-1-\beta)}\right)}{a\left(v_{\sigma^{\beta}(l)}^{(n-\beta)}, v_{\sigma^{\beta}(k)}^{(n-\beta)}\right)}\right. \\
& \times \prod_{\theta=1}^{\beta}\left(B_{p_{1}}^{*}\left(v_{\sigma^{\theta}(1)}^{(n-\theta)}, \ldots, v_{\sigma^{\theta}\left(p_{1}\right)}^{(n-\theta)} \mid v_{\sigma^{\theta+1}(1)}^{(n-1-\theta)}, \ldots, v_{\sigma^{\theta+1}\left(p_{1}\right)}^{(n-1-\theta)}\right)\right. \\
& \left.\left.\left.\times \prod_{\gamma=2}^{\theta} B_{p_{\gamma}-p_{\gamma-1}}\left(v_{\sigma^{\theta}\left(p_{\gamma-1}+1\right.}^{(n-\theta)}\right), \ldots, v_{\sigma^{\theta}\left(p_{\gamma}+1\right)}^{(n-\theta)} \mid v_{\sigma^{\theta+1}\left(p_{\gamma-1}+1\right)}^{(n-1-\theta)}, \ldots, v_{\sigma^{\theta+1}\left(p_{\gamma}+1\right)}^{(n-1-\theta)}\right)\right)\right]
\end{aligned}
$$

and for $m>1$,

$$
\begin{aligned}
& B_{n_{1},\left(p_{1}, \ldots, p_{m+n-2}\right)}^{(0)}\left(v_{1}, \ldots, v_{n_{1}} ; v_{1}^{(1)}, \ldots, v_{p_{1}}^{(1)}, \ldots, v_{p_{m+n-2}}^{(1)} \mid z_{i_{1}}, \ldots, z_{i_{n_{1}}}\right) \\
& =B_{p_{1}}^{* *}\left(v_{1}^{(m+n-2)}, \ldots, v_{p_{1}}^{(m+n-2)} \mid v_{\sigma^{2}(1)}^{(m+n-3)}, \ldots, v_{\sigma^{2}\left(p_{1}\right)}^{(m+n-3)}\right) \\
& \times \prod_{\beta=2}^{m-1}\left[\sum_{\sigma^{\beta} \in \mathcal{S}_{p_{\beta}}}\left(\prod_{\epsilon=0}^{\beta-1} \prod_{\sigma^{\beta}(j)>\sigma^{\beta}(i)=p_{\epsilon}+1}^{p_{\epsilon+1}}\left(1+c_{\sigma^{\beta}(i) \sigma^{\beta}(j)}\right)\right)^{-1}\right. \\
& \times \prod_{\alpha=1}^{\beta-1} \prod_{k=1}^{p_{\alpha}} \prod_{l=p_{\alpha}+1}^{p_{\alpha}} \frac{c\left(v_{\sigma^{\beta}(l)}^{(m+n-1-\beta)}, v_{\sigma^{\beta}(k)}^{(m+n-1-\beta)}\right) a\left(v_{\sigma^{\beta}(l)}^{(m+n-1-\beta)}, v_{\sigma^{\beta+1}\left(k_{\alpha}\right)}^{(m+n-2-\beta)}\right)}{a\left(v_{\sigma^{\beta}(l)}^{(m+n-1-\beta)}, v_{\sigma^{\beta}(k)}^{(m+n-1-\beta)}\right)} \\
& \left.\times \prod_{\theta=2}^{\beta} \prod_{\alpha=1}^{\theta} B_{p_{\alpha}-p_{\alpha-1}}^{* *}\left(v_{\sigma^{\theta}\left(p_{\alpha-1}+1\right)}^{(m+n-1-\theta)}, \ldots, v_{\sigma^{\theta}\left(p_{\alpha}\right)}^{(m+n-1-\theta)} \mid v_{\sigma^{\theta+1}\left(p_{\alpha-1}+1\right)}^{(m+n-2-\theta)}, \ldots, v_{\sigma^{\theta+1}\left(p_{\alpha}\right)}^{(m+n-2-\theta)}\right)\right] \\
& \times \prod_{\beta=m}^{m+n-1}\left[\sum_{\sigma^{\beta} \in \mathcal{S}_{p_{\beta}}} \operatorname{Exp}\left\{\delta_{\beta, m} \ln c_{1, \ldots p_{\beta}}^{\sigma^{\beta}}\right\}\left(\prod_{\epsilon=0}^{m-1} \prod_{\sigma^{\beta}(j)>\sigma^{\beta}(i)=p_{\epsilon}+1}^{p_{\epsilon+1}}\left(1+c_{\sigma^{\beta}(i) \sigma^{\beta}(j)}\right)\right)^{-1}\right.
\end{aligned}
$$




$$
\begin{aligned}
& \times \prod_{\alpha=1}^{m-1} \prod_{k=1}^{p_{\alpha}} \prod_{l=p_{\alpha}+1}^{p_{\alpha+1}}\left(-\frac{a\left(v_{\sigma^{\beta}(l)}^{(m+n-1-\beta)}, v_{\sigma^{\beta+1}\left(k_{\alpha}\right)}^{(m+n-2)}\right)}{a\left(v_{\sigma^{\beta}(l)}^{(m+n-1-\beta)}, v_{\sigma^{\beta}(k)}^{(m+n-1-\beta)}\right)}\right) \\
& \times \prod_{\alpha=m}^{m} \prod_{k=1}^{m} \prod_{l=p_{\alpha}+1}^{p_{\alpha+1}} \frac{a\left(v_{\sigma^{\beta}(l)}^{(m+n-1-\beta)}, v_{\sigma^{\beta+1}\left(k_{\alpha}\right)}^{(m+n-2)}\right)}{a\left(v_{\sigma^{\beta}(l)}^{(m+n-1-\beta)}, v_{\sigma^{\beta}(k)}^{(m+n-1-\beta)}\right)} \\
& \times \prod_{\theta=m}^{\beta}\left(\prod_{\alpha=1}^{m} B_{p_{\alpha}-p_{\alpha-1}}^{*}\left(v_{\sigma^{\theta}\left(p_{\alpha-1}+1\right)}^{(m+n-1) \theta)}, \ldots, v_{\sigma^{\theta}\left(p_{\alpha}\right)}^{(m+n-1-\theta)} \mid v_{\sigma^{\theta+1}\left(p_{\alpha-1}+1\right)}^{(m+n-2) \theta)}, \ldots, v_{\sigma^{\theta+1}\left(p_{\alpha}\right)}^{(m+n-2)}\right)\right. \\
& \left.\left.\times \prod_{\gamma=m+1}^{\theta} B_{p_{\gamma}-p_{\gamma-1}}\left(v_{\sigma^{\theta}\left(p_{\gamma-1}+1\right)}^{(m+n-1-\theta)}, \ldots, v_{\sigma^{\theta}\left(p_{\gamma}\right)}^{(m+n-1-\theta)} \mid v_{\sigma^{\theta+1}\left(p_{\gamma-1}+1\right)}^{(m+n-2-\theta)}, \ldots, v_{\sigma^{\theta+1}\left(p_{\gamma}\right)}^{(m+n-2)-\theta)}\right)\right)\right]
\end{aligned}
$$

with the conventions $p_{0}=0, p_{m+n-1}=n_{1}, v^{(0)}=v, v^{(-1)}=u, \sigma^{m+n}=1$, and $B_{n}^{* *}\left(v_{1}^{(l)}, \ldots, v_{n}^{(l)} \mid v_{1}^{(l-1)}, \ldots, v_{n}^{(l-1)}\right)=B_{n}^{(2)}\left(v_{1}^{(l)}, \ldots, v_{n}^{(l)} \mid v_{l-1}^{(1)}, \ldots, v_{n}^{(l-1)}\right)$.

\section{Discussions}

In this paper, we have constructed the factorizing $F$-matrices for the $g l(m \mid n)$-invariant fermion model. In the basis provided by the $F$-matrix (the $F$-basis), the monodromy matrix and the creation operators take completely symmetric forms. We moreover have obtained a symmetric representation of the Bethe vector of the system.

Authors in [19] derived a formula that expresses the local spin and field operators of fundamental graded models in terms of the elements of the monodromy matrix. In particular they reconstructed the local operators $\left(E^{i j}\right)$ in terms of operators figuring in the $g l(m \mid n)$ monodromy matrix. This together with the results of the present paper in the $F$-basis should enable one to get the exact representations of form factors and correlation functions of the supersymmetric fermion models. These are under investigation and results will be reported elsewhere.

Acknowledgements: This work was financially supported by the Australia Research Council. S.Y. Zhao has also been supported by the UQ Postdoctoral Research Fellowship.

\section{References}

[1] J. M. Maillet and I. Sanchez de Santos, Drinfel'd twists and algebraic Bethe ansatz, q-alg/9612012.

[2] V. G. Drinfeld, Constant quasi-classical solutions of the Yang-Baxter quantum equation, Sov. Math. Dokl. 28 (1983) 667. 
[3] N. Kitanine, J.M. Maillet and V. Terras, Form factors of the XXZ Heisenberg spin-1/2 finite chain, Nucl. Phys. B554 (1999) 647-678; math-ph/9807020.

[4] A. G. Izergin, N. Kitanine, J. M. Maillet and V. Terras, Spontaneous magnetization of the XXZ Heisenberg spin-1/2 chain, Nucl. Phys. B554 (1999) 679-696; solv-int/9812021

[5] V. Terras, Drinfel'd twists and functional Bethe ansatz, Lett. Math. Phys. 48 (1999) 263-276; math-ph/9902009.

[6] T.-D. Albert, H. Boos, R. Flume and K. Ruhlig, Resolution of the nested hierarchy for the rational sl(n) models, J. Phys. A33 (2000) 4963-4980; nlin.SI/0002027.

[7] T.-D. Albert, H. Boos, R. Flume, R.H. Poghossian and K. Ruhlig, An F-twisted XYZ model, Lett. Math. Phys. 53 (2000) 201-214; nlin.SI/0005023.

[8] T.-D. Albert and K. Ruhlig, Polarization free generators for the Belavin model, J. Phys. A34 (2001) 1569-1580; nlin.SI/0007020.

[9] J.H.H. Perk and C.L. Schultz, New families of commuting transfer matrices in q-state vertex models, Phys. Lett. A84 (1981) 407-410.

[10] C.L. Schultz, Eigenvectors of the multi-component generalization of the six-vertex model, Physica A122 (1983) 71.

[11] H. J. De Vega and E. Lopes, Exact solution of the Perk-Schultz model, Phys. Rev. Lett. 67 (1991) 489-492.

[12] E. Lopes, Exact solution of the multi-component generalized six-vertex model, Nucl. Phys. B370 (1992) 636-658.

[13] P.P. Kulish and E.K. Sklyanin, On solutions of the Yang-Baxter equation, Zap. Nauch. Semin. LOMI 95 (1980) 129-160 (English translation: J. Sov. Math. 19 (1982) 1596).

[14] F.H.L. Essler, V.E. Korepin and K. Schoutens, Exact solution of an electronic model of supercondutivity, Int. J. Mod. Phys. B23 (1994) 3205-3242; cond-mat/9211001.

[15] A.J. Bracken, M.D. Gould, J.R. Links and Y.-Z. Zhang, A new supersymmetric and exactly solvable model of correlated electrons, Phys. Rev. Lett. 74 (1995) 2768-2771; cond-mat/9410026.

[16] P.P. Kulish, Integrable graded magnets, Zap. Nauch. Semin. LOMI 145 (1985) 140-163 (English translation: J. Sov. Math. 35 (1986) 2648).

[17] W.-L. Yang, Y.-Z. Zhang and S.-Y. Zhao, Drinfeld twists and algebraic Bethe ansatz of the supersymmetric $t$ - $J$ model, J. High Energy Physics (JHEP) 12 (2004) 038 (24 pages); cond-mat/0412182. 
[18] W.-L. Yang, Y.-Z. Zhang and S.-Y. Zhao, Drinfeld twists and algebraic Bethe ansatz of the quantum supersymmetric model associated with $U_{q}(g l(m \mid n))$, eprint: hep-th/0503003.

[19] F. Göhmann and V. E. Korepin, Solution of the quantum inverse problem, J. Phys. A33 (2000) 1199-1220; hep-th/9910253 